\title{
SOME EXAMPLES FOR THE POINCARÉ AND PAINLEVÉ PROBLEMS
}

\author{
BY AlCIDES LINS NETO ${ }^{1}$
}

ABSTRACT. - In 1891, Poincaré started a series of three papers in which he tried to answer the following question (cf. [21-23]): "Is it possible to decide if an algebraic differential equation in two variables is algebraically integrable?" (in the sense that it has a rational first integral). More or less at the same time P. Painlevé asked the following question: "Is it possible to recognize the genus of the general solution of an algebraic differential equation in two variables which has a rational first integral?". In this paper we give examples of one-parameter families which show that both problems have a negative answer. With some of the families we can also answer a question posed by M. Brunella in [5].

(C) 2002 Éditions scientifiques et médicales Elsevier SAS

RÉSUMÉ. - En 1891, Poincaré commençait une série de trois articles dans lesquels il tentait de répondre à la question suivante (cf. [21-23]) : «Est-il possible de décider si une équation différentielle algébrique en deux variables est algébriquement intégrable ?» (au sens où il admet une intégrale première rationnelle). À peu près à la même époque, P. Painlevé posait la question suivante : «Est-il possible de déterminer le genre de la solution générale d'une équation différentielle algébrique en deux variables qui admet une intégrale première rationnelle ?». Dans cet article, nous donnons des exemples de familles à un paramètre qui montrent que les deux problèmes ont une réponse négative. À l'aide de certaines de ces familles, nous pouvons également répondre à une question de M. Brunella dans [5].

(c) 2002 Éditions scientifiques et médicales Elsevier SAS

\section{Introduction}

The main purpose of this paper is to describe some examples of one-parameter families of algebraic differential equations on two variables which show that the so-called "Poincaré problem" and "Painlevé problem" have a negative answer. With some of the families we give an answer for a problem stated by M. Brunella in [5].

H. Poincaré in three papers [21,22] and [23], studied the following problem:

"Is it possible to decide if an algebraic differential equation in two variables has a rational first integral?"

In [22] he starts, by observing that it is sufficient to bound the degree of a possible algebraic solution. In fact, in [22] and [23] he tries to bound this degree, by supposing that the equation has a fixed degree (he calls "dimension" of the equation, its degree) and non-degenerate singularities of fixed local analytic type, that is, with local first integral of the type $u^{p} \cdot v^{q}=c t e$, where $p$

\footnotetext{
${ }^{1}$ This research was partially supported by Pronex. 
and $q$ are non zero relatively primes integers which depend of the singularity. When $p>0$ and $q<0$, the equation has a local meromorphic first integral and he calls the singularity "dicritical" or "node" ("nœud"). When $p, q>0$ the equation has a local holomorphic first integral and he calls the singularity a "saddle" ("col"). In [21] he announces the results that he proves in [22]. In particular, he solves the problem in some particular cases like, for instance, in the case where in all the saddles we have $p / q=-1$. He also observes that this problem was studied twenty years before by Darboux (cf. [11]) and also by Painlevé and Autonne more or less at the same time than himself. Another problem, posed by P. Painlevé, is the following (cf. [20] and [21]):

"Is it possible to recognize the genus of the general solution of an algebraic differential equation in two variables which has a rational first integral?"

As we will see, in the families constructed in the begining, all differential equations with rational first integral have their general integral curve of genus one. However, by pulling-back these examples by rational maps, it is possible to construct families which contain differential equations with general integral curves of arbitrarily large genus.

More recently the problem of bounding the degree of a solution, was studied by several authors in a more general context, that is, without the hypothesis that the equation has a rational first integral. In particular, it was solved in some particular cases, where conditions are imposed either on the solution or on the singular points of the equation (cf. [9,8] and [7]).

Before stating our results, we will recall some basic notions which will be used along the text. We will use freely some basic facts and the terminology of the theory of foliations (leaf, holonomy, etc...) (cf. [13] and [6]). Given a vector field $X=P(x, y) \frac{\partial}{\partial x}+Q(x, y) \frac{\partial}{\partial y}$, where $P$ and $Q$ are polynomials on $\mathbb{C}^{2}$, the singular set of $X$ is, by definition, the algebraic set $\operatorname{sing}(X)=\left\{(x, y) \in \mathbb{C}^{2} ; P(x, y)=Q(x, y)=0\right\}$. In this paper we will suppose that $\operatorname{sing}(X)$ is finite (i.e., $P$ and $Q$ are relatively prime polynomials). A singular point $p_{o}=\left(x_{o}, y_{o}\right)$ of $X$ is non-degenerate if the jacobian matrix $D X\left(p_{o}\right)$ is non singular. In this case, if $\lambda_{1}$ and $\lambda_{2}$ are the eigenvalues of $D X\left(p_{o}\right)$, then the quotients $\lambda_{1} / \lambda_{2}$ and $\lambda_{2} / \lambda_{1}$ are called the characteristic numbers of the singularity. These characteristic numbers are analytic invariants of the singularity (cf. [2] or [18]).

Outside $\operatorname{sing}(X)$, the system of complex differential equations associated to $X$ :

$$
\frac{d x}{d t}=P(x, y) \quad \frac{d y}{d t}=Q(x, y)
$$

generates a foliation of $\mathbb{C}^{2}$, whose leaves are the images of the complete solutions of (1), that is, the immersed Riemann surfaces on $\mathbb{C}^{2}$ locally parametrized by the solutions of (1). Since $P$ and $Q$ are polynomials, this foliation can be extended to a unique singular foliation on the projective space $\mathbb{C} P(2)$, say $\mathcal{F}$. We will say that the vector field $X$ represents $\mathcal{F}$ in the affine chart $\mathbb{C}^{2} \subset \mathbb{C P}(2)$. The degree of $\mathcal{F}$ is, by definition, the number of tangencies of $\mathcal{F}$ with a generic line, linearly embeded in $\mathbb{C} P(2)$. If $\mathcal{F}$ is of degree $d$ then the vector field $X$ is of the form

$$
X=[p(x, y)+x . g(x, y)] \frac{\partial}{\partial x}+[q(x, y)+y \cdot g(x, y)] \frac{\partial}{\partial y},
$$

where $p, q$ are polynomials of degree $\leqslant d$ and $g$ is a homogeneous polynomial of degree $d$, such that, if $g \equiv 0$ then $\max \{d g(p), d g(q)\}=d$ and the homogeneous part of $p$ and $q$ of degree $d$ are not of the form $x . h$ and $y . h$, respectively. When all singularities of $\mathcal{F}$ are non-degenerate, then $\mathcal{F}$ has exactly $d^{2}+d+1$ singularities (where in this number we count also the singularities which appear at line of infinite after the compactification). This formula is also true when all singularities of $\mathcal{F}$ are isolated, if we count the singularities with multiplicity (cf. [17]). 
We say that an algebraic curve $C$ is invariant by $\mathcal{F}$, or a solution of $\mathcal{F}$, if it is the union of leaves and singularities of $\mathcal{F}$. For instance, the line at infinity of $\mathbb{C}^{2}, L=\mathbb{C} P(2) \backslash \mathbb{C}^{2}$, is an algebraic solution of $\mathcal{F}$ if and only if $g \equiv 0$ in (2). More generally, if $C \neq L$ is given in the affine coordinate system $\mathbb{C}^{2}$ by $(f=0)$, where $f$ is a reduced polynomial, then $C$ is a solution of $\mathcal{F}$ if, and only if, $X(f):=P \frac{\partial f}{\partial x}+Q \frac{\partial f}{\partial y}=f . h$, where $h$ is some polynomial (cf. [17]). The foliation $\mathcal{F}$ has a rational first integral (or is algebraically integrable) if there exists a rational function $F: \mathbb{C} P(2)-\rightarrow \overline{\mathbb{C}}$ such that all level curves of $F$ are solutions of $\mathcal{F}$.

Another way of representing the foliation $\mathcal{F}$ defined by (1) in $\mathbb{C}^{2}$, is by the differential equation $\omega=0$, where $\omega=P(x, y) d y-Q(x, y) d x$. When we represent $\mathcal{F}$ in this way, then the rational function $F$ is a first integral of $\mathcal{F}$ if, and only if, $d F=H . \omega$, where $H$ is a rational function. Since $F$ is a quotient of polynomials, say $F=g / h$, then this condition is equivalent to

$$
h . d g-g . d h=r . \omega,
$$

where $r$ is some polynomial. One of the main difficulties of the problem to recognize if a differential equation is algebraically integrable or not, is that, although the degree of $\omega$ is fixed, the degrees of $g, h$ and $r$ are, in principle, arbitrary. If we could, in some way, bound the degree of a possible algebraic solution of (1), then the existence of $g, h$ and $r$ like in (3) would be reduced to an algebraic system of equations involving the (unknown) coefficients of these three polynomials and the (known) coefficients of $P$ and $Q$.

Example. - We say that a foliation $\mathcal{F}$ in $\mathbb{C P}(2)$ has a Darboux first integral if it can be defined in an affine chart by a differential equation $\omega=f . \eta=0$, where $f$ is a rational function and $\eta$ is a closed meromorphic form. It can be shown that $\eta$ must be of the following type:

$$
\eta=\sum_{j=1}^{r} \lambda_{j} \frac{d f_{j}}{f_{j}}+d g
$$

where $f_{1}, \ldots, f_{r}$ are polynomials and $g$ is a rational function (cf. [10]). The Darboux integral is $f_{1}^{\lambda_{1}} \cdots f_{r}^{\lambda_{r}} \cdot e^{g}$, in this case.

A simple example in which a family of differential equations of some fixed degree contains members with first integrals of arbitrarily large degrees is given by a family like above, when $g=0$. Consider the family of foliations in $\mathbb{C} P(2)$ given in an affine chart by the differential equations

$$
\omega_{\lambda}:=f_{1} \cdots f_{r} \sum_{j=1}^{r} \lambda_{j} \frac{d f_{j}}{f_{j}}=0,
$$

where $r \geqslant 2, \lambda=\left(\lambda_{1}, \ldots, \lambda_{r}\right) \in\left(\mathbb{C}^{*}\right)^{r}$ and $f_{1}, \ldots, f_{r}$ are polynomials in $\mathbb{C}^{2}$. The foliation $\mathcal{F}\left(\omega_{\lambda}\right)$, defined by (4) in $\mathbb{C P}(2)$, has a meromorphic first integral if, and only if, $\lambda=c . m$ where $m=\left(m_{1}, \ldots, m_{r}\right) \in \mathbb{Z}^{r}$ and $c \in \mathbb{C}^{*}$. However, in this family the singularities of $\mathcal{F}\left(\omega_{\lambda}\right)$ are not of fixed analytic type. In fact, suppose that $r \geqslant 2$ and that $f_{1}, \ldots, f_{r}$ are chosen in such a way that there is a point $p \in\left(f_{1}=f_{2}=0\right) \backslash \bigcup_{j \geqslant 3}\left(f_{j}=0\right)$, which is not a singularity of the curves $\left(f_{1}=0\right)$ and $\left(f_{2}=0\right)$, and that these two curves meet transversely at $p$. In this case, a simple calculation shows that $\omega_{\lambda}(p)=0$, that this singularity is non-degenerate and their characteristic numbers are $-\lambda_{1} / \lambda_{2}$ and $-\lambda_{2} / \lambda_{1}$.

The above example shows that, in order to bound the degree of a possible algebraic solution, we must fix the analytic type of the singularities of the foliation. 
DEFINITION 1. - Let $\left(\mathcal{F}_{s}\right)_{s \in S}$ be a family of algebraic foliations on $\mathbb{C} P(2)$, where $S$ is some complex manifold of parameters and the coefficients of the differential equations which define the family in some fixed affine chart depend holomorphically of $s \in S$. We say that the family has singularities of fixed analytic type, if

(i) The set of singularities of $\mathcal{F}_{s}, s \in S$, can be written as $\operatorname{sing}\left(\mathcal{F}_{s}\right)=\left\{p_{1}(s), \ldots, p_{k}(s)\right\}$, where the functions $s \in S \mapsto p_{j}(s)$ are holomorphic.

(ii) For each $j \in\{1, \ldots, k\}$ and $s_{1}, s_{2} \in S$, there exists a biholomorphism $g$ from a neighborhood $U_{1}$ of $p_{j}\left(s_{1}\right)$ to a neighborhood $U_{2}$ of $p_{j}\left(s_{2}\right)$ which sends $p_{j}\left(s_{1}\right)$ to $p_{j}\left(s_{2}\right)$ and leaves of $\mathcal{F}_{s_{1}}$ in $U_{1}$ onto leaves of $\mathcal{F}_{s_{2}}$ in $U_{2}$. In particular, if the singularity $p_{j}\left(s_{1}\right)$ is non-degenerate for $\mathcal{F}_{s_{1}}$ then $p_{j}\left(s_{2}\right)$ is also non-degenerate for $\mathcal{F}_{s_{2}}$ and they have the same characteristic numbers. When all singularities of $\mathcal{F}_{s}$ are non-degenerate (for all $s \in S$ ), we will say that the family has non-degenerate singularities.

Our main result is the following:

MAIN THEOREM. - For $d=2,3,4$, there are families of foliations in $\mathbb{C P}(2)$, say $\left(\mathcal{F}_{\alpha}^{d}\right)_{\alpha \in \overline{\mathbb{C}}}$, of degree $d$, with the following properties:

(a) There is a finite set of parameters $A^{d} \subset \overline{\mathbb{C}}$ such that the restricted family $\left(\mathcal{F}_{\alpha}^{d}\right)_{\alpha \in \overline{\mathbb{C}} \backslash A^{d}}$ has non-degenerate singularities of fixed analytic type.

(b) There exists a countable and dense set of parameters $E \subset \overline{\mathbb{C}}$, such that for any $\alpha \in E$ the foliation $\mathcal{F}_{a}^{d}$ has a rational first integral, say $F_{\alpha}=P_{\alpha} / Q_{\alpha}$, of degree $d_{\alpha}$ (that is the general level curve $F_{\alpha}=$ cte is irreducible of degree $d_{\alpha}$ ), satisfying the property that for any $k>0$ the set $\left\{\alpha \in E ; d_{\alpha} \leqslant k\right\}$ is finite. In particular, the degrees of the rational first integrals can be chosen arbitrarily large and these families provide counterexample for the Poincaré problem.

(c) If $\alpha \notin E$, then $\mathcal{F}_{\alpha}^{d}$ has no rational first integral. In particular, almost all leaves of $\mathcal{F}_{\alpha}^{d}$ are transcendent (non-algebraic). Furthermore, for any of these transcendent leaves, say $L$, there exists a holomorphic non-constant map $f: \mathbb{C} \rightarrow \mathbb{C} P(2)$, such that $f(\mathbb{C}) \subset$ $L \cup \operatorname{sing}\left(\mathcal{F}_{\alpha}^{d}\right)$.

Remark 1. - For the foliations like in (b) of the theorem, almost all levels $F_{\alpha}=$ cte are elliptic curves, that is are curves of genus one (see Proposition 6 of \$2.2). Therefore these families are not couterexamples for the Painlevé problem. However, by pulling-back such families by rational maps $\Phi: \mathbb{C} P(2) \rightarrow \mathbb{C} P(2)$ of topological degree $>1$, we can obtain such couterexamples, as in the following:

COROLlaRY. - For any $d \geqslant 5$, there exists a family of foliations of degree $d$ in $\mathbb{C P}(2)$, say $\left(\mathcal{F}_{\alpha}^{d}\right)_{\alpha \in \overline{\mathbb{C}}}$, satisfying (a) of the main Theorem and

(b.1) There exists a countable and dense set of parameters $E \subset \overline{\mathbb{C}}$, such that for any $\alpha \in E$ the foliation $\mathcal{F}_{a}^{d}$ has a rational first integral, say $F_{\alpha}$, of degree $d_{\alpha}$, satisfying the property that for any $k>0$ the sets $\left\{\alpha \in E^{d} ; d_{\alpha} \leqslant k\right\}$ and

$$
\left\{\alpha \in E ; \text { such that the genus of the general level curve of } F_{\alpha} \text { is } \leqslant k\right\}
$$

are finite. In particular, the degrees of the rational first integrals and the genus of their general level curves can be chosen arbitrarily large and these families provide couterexamples for Painlevé problem.

Remark 2. - As we will see, the singularities of the foliations in $\mathcal{F}_{\alpha}^{d}, \alpha \notin A^{d}$, are singularities with local first integrals of the following analytic types:

(a.1) In the degree four situation: $v / u=c t e$ for the dicritical singularities and $v^{3} \cdot u=c t e$ for the saddles. 
(a.2) In the degree three situation: $v / u=c t e$ or $v^{2} / u=c t e$ for the dicritical singularities and $v^{3} \cdot u=$ cte or $v^{6} \cdot u=$ cte for the saddles.

(a.3) In the degree two situation: $v^{2} / u=c t e$ or $v^{3} / u^{2}=$ cte for the dicritical singularities and $v^{3} \cdot u=c t e$ or $v^{6} \cdot u=c t e$ for the saddles.

Remark 3. - At the end of $\$ 2.2$ we will prove that, for the foliations $\mathcal{F}_{\alpha}^{4}$ in the main Theorem, $\alpha \notin E$, after the resolution of the dicritical singularities of $\mathcal{F}_{a}^{4}$, almost all leaves of the resolved foliation are biholomorphic to $\mathbb{C}$. We observe that these foliations answer a question of M. Brunella in [5]. In [5], Brunella proved that, if $\mathcal{F}$ is a foliation in $\mathbb{C P}(2)$ of degree $\geqslant 5$, whose singularities are non-degenerate, and if $f: \mathbb{C} \rightarrow \mathbb{C P}(2)$ is a non-constant holomorphic map tangent to $\mathcal{F}$, then $f(\mathbb{C})$ must be contained in an algebraic solution of $\mathcal{F}$. At the end of [5] he asks if the same result could be proved for foliations of degree $\geqslant 2$.

Remark 4. - In the main Theorem and in the Corollary, when $\alpha \notin E$ the foliation $\mathcal{F}_{\alpha}^{d}$ is not Darboux integrable. On the other hand, it is Liouvillian integrable, that is, the foliation $\mathcal{F}_{\alpha}^{d}$ can be defined by a meromorphic 1 -form $\omega_{\alpha}$ in $\mathbb{C P}(2)$ in such a way that there exists a meromorphic closed 1-form $\eta$ such that $d \omega_{\alpha}=\eta \wedge \omega_{\alpha}$. This fact will be proved for the family of degree four in Remark 6 of $\$ 2.2$. We would like to observe also that, since the families are not Darboux integrable, the set $E$ cannot be described explicitly (in principle). It would be interesting to know what kinds of properties this set has.

Remark 5. - Another interesting fact about any of the families is that, if $\alpha \notin E$ then:

(i) For any $p \in \mathbb{C P}(2)$, there exists a neighborhood $U$ of $p$ such that $\left.\mathcal{F}_{\alpha}^{d}\right|_{U}$ has a first integral (holomorphic or meromorphic).

(ii) $\mathcal{F}_{\alpha}^{d}$ has no meromorphic global first integral.

As far as we know, these are the first known examples of algebraic foliations in $\mathbb{C P}(2)$ with such properties.

We would like to state some problems which arise naturally from the examples and some well-known results.

Problem 1.- An useful result, due to Darboux and later generalized by Jouanolou, is the following (cf. [14] (p. 29), [11] and [15]):

THEOREM. - For all $d \geqslant 1$ there exists $N(d) \in \mathbb{N}$ such that if a foliation of degree $d$ has more than $N(d)$ algebraic solutions, then it has a rational first integral. In particular, a foliation with infinitely many algebraic solutions has a rational first integral.

A natural question should be the following: given $d \geqslant 2$, is there $M(d) \in \mathbb{N}$ such that if a foliation of degree $d$ has an algebraic solution of degree greater than or equal to $M(d)$, then it has a rational first integral? However, I recently have received a preprint by J. Moulin Ollagnier [19] in which he claims that this question has a negative answer, at least for foliations of degree two. In this paper he exhibits a countable family of Lotka-Volterra polynomial differential equations, say $L V(\ell), \ell \in \mathbb{N}$, without rational first integrals, with associated foliations of degree two, and such that $L V(\ell)$ has an irreducible algebraic solution of degree $2 \ell$. We observe that the singularities of the foliations in Ollagnier's family are not of fixed analytic type. On the other hand, all such foliations have Liouvillian first integrals, so that a more realistic question would be the following:

Question. - Given $d \geqslant 2$, is there $M(d) \in \mathbb{N}$ such that if a foliation of degree $d$ has an algebraic solution of degree greater than or equal to $M(d)$, then it has a Liouvillian first integral?

Problem 2. - Classify all families of foliations in $\mathbb{C P}(2)$ having properties (a), (b) and (c) of the families of the main Theorem. 
Problem 3. - Classify all families of foliations in $\mathbb{C P}(2)$ having properties (a) and (b.1) of the families of the Corollary.

I would like to thank the referee for helpful ideas and suggestions which enabled me to simplify and improve some of the steps of the proofs.

\section{Proof of the main Theorem}

We begin by describing a configuration of lines and points in $\mathbb{C P}(2)$ that will be used in the construction of the family of degree four.

\subsection{A particular configuration of lines and points}

Let us consider the lines in $\mathbb{C P}(2)$ defined in homogeneous coordinates by the equation

$$
\left(Y^{3}-X^{3}\right)\left(Z^{3}-Y^{3}\right)\left(X^{3}-Z^{3}\right)=0 .
$$

It is not difficult to see that the nine lines defined by (5), say $\ell_{1}, \ldots, \ell_{9}$, intersect in twelve points of $\mathbb{C P}(2)$, which we will denote by $p_{1}, \ldots, p_{12}$. We will use the notation $j$ for the primitive cubic root of the unity $\mathrm{e}^{2 \pi \mathrm{i} / 3}$.

In Fig. 1 we sketch the configuration in the affine plane $(Z=1)$. The lines $\ell_{1}, \ldots, \ell_{9}$ in this case are represented by $(x=1),(x=j),\left(x=j^{2}\right),(y=1),(y=j),\left(y=j^{2}\right),(y=x)$, $(y=j x)$ and $\left(y=j^{2} x\right)$. The points of the configuration in the finite plane are $(0,0)$ and $\left(j^{k}, j^{\ell}\right)$, $k, \ell \in\{0,1,2\}$, and the two points $[1: 0: 0]$ and $[0: 1: 0]$ at the line $(Z=0)$. We will denote this configuration by $\mathcal{C}=(\mathcal{L}, \mathcal{P})$, where $\mathcal{L}=\left\{\ell_{1}, \ldots, \ell_{9}\right\}$ and $\mathcal{P}=\left\{p_{1}, \ldots, p_{12}\right\}$.

We observe that the configuration $\mathcal{C}$ satisfies the following properties:

(I) Each line contains four points of the configuration.

(II) Each point belongs to three lines of the configuration.

(III) If three points of $\mathcal{P}$ are not in a line $\ell \in \mathcal{L}$, then the points are not aligned.

In the next proposition we obtain some symmetries of the configuration $\mathcal{C}$ and prove that it is essentially the unique configuration satisfying (I), (II) and (III).

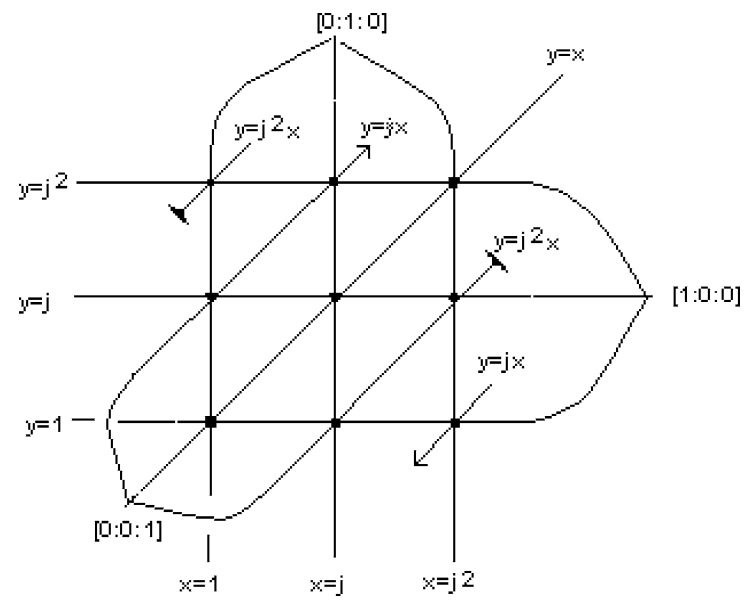

Fig. 1. 
Proposition 1. - Let $\mathcal{C}^{\prime}=\left(\mathcal{L}^{\prime}, \mathcal{P}^{\prime}\right)$ be a configuration of nine lines and twelve points, where $\mathcal{L}^{\prime}=\left\{\ell_{1}^{\prime}, \ldots, \ell_{9}^{\prime}\right\}$ and $\mathcal{P}^{\prime}=\left\{p_{1}^{\prime}, \ldots, p_{12}^{\prime}\right\}$ are the sets of lines and points of $\mathcal{C}^{\prime}$. Suppose that $\mathcal{C}^{\prime}$ satisfies properties (I), (II) and (III) above. Then $\mathcal{P}^{\prime}$ can be divided into four subsets $P_{1}, \ldots, P_{4}$, each one containing three points, with the following properties:

(a) $P_{i} \cap P_{j}=\emptyset$ if $i \neq j$.

(b) If $P_{j}=\left\{p_{1}^{\prime}, p_{2}^{\prime}, p_{3}^{\prime}\right\}$, then there exists an automorphism $T$ of $\mathbb{C P}(2)$ which sends $\mathcal{C}^{\prime}$ into $\mathcal{C}\left(\right.$ that is $T\left(\mathcal{L}^{\prime}\right)=\mathcal{L}$ and $\left.T\left(\mathcal{P}^{\prime}\right)=\mathcal{P}\right)$ in such a way that $T\left(p_{1}^{\prime}\right)=[0: 0: 1]$, $T\left(p_{2}^{\prime}\right)=[0: 1: 0]$ and $T\left(p_{3}^{\prime}\right)=[1: 0: 0]$.

(c) Given $i, j \in\{1, \ldots, 4\}, i \neq j$, there exists an automorphism $S$ of $\mathbb{C P}(2)$ such that $S\left(\mathcal{C}^{\prime}\right)=\mathcal{C}^{\prime}$ and $S\left(P_{i}\right)=P_{j}$.

(d) Given $p_{i}^{\prime}, p_{j}^{\prime} \in \mathcal{P}$, there exists an automorphism $S$ of $\mathbb{C P}(2)$ such that $S\left(\mathcal{C}^{\prime}\right)=\mathcal{C}^{\prime}$ and $S\left(p_{i}^{\prime}\right)=p_{j}^{\prime}$.

Proof. - Choose a point $p_{1}^{\prime} \in \mathcal{P}^{\prime}$. It follows from (II) that $p_{1}^{\prime}$ belongs to three lines, say $\ell_{1}^{\prime}, \ell_{2}^{\prime}$ and $\ell_{3}^{\prime}$. Since each of these lines contain four points of $\mathcal{P}^{\prime}$, it is clear that $\ell_{1}^{\prime} \cup \ell_{2}^{\prime} \cup \ell_{3}^{\prime}$ contains ten points of $\mathcal{P}^{\prime}$, so that there are two other points in $\mathcal{P}^{\prime}$, which we can suppose to be $p_{2}^{\prime}$ and $p_{3}^{\prime}$. Let $P_{1}=\left\{p_{1}^{\prime}, p_{2}^{\prime}, p_{3}^{\prime}\right\}$. If we consider now a point $p_{i}^{\prime} \in \mathcal{P}^{\prime} \backslash P_{1}$, then we can define in a similar way a subset $P_{2}$, with three points, such that $p_{i}^{\prime} \in P_{2}, P_{1} \cap P_{2}=\emptyset$ and the other two points of $P_{2}$ are not contained in the three lines of $\mathcal{L}^{\prime}$ which contain $p_{i}^{\prime}$.

In the same way, we can define subsets $P_{3}$ and $P_{4}$ (inductively), such that $P_{1}, \ldots, P_{4}$ satisfy (a) of the proposition and for each $j \in\{1, \ldots, 4\}$ the three points of $P_{j}$ are not aligned and two of them do not belong to the same line of $\mathcal{L}^{\prime}$. We leave the details for the reader.

Now, let us consider $P_{1}=\left\{p_{1}^{\prime}, p_{2}^{\prime}, p_{3}^{\prime}\right\}$ and prove that it satisfies (b). Since $p_{1}^{\prime}, p_{2}^{\prime}$ and $p_{3}^{\prime}$ are not aligned, there exists an automorphism $T_{1}$ of $\mathbb{C P}(2)$ such that $T_{1}\left(p_{1}^{\prime}\right)=[0: 0: 1]$, $T_{1}\left(p_{2}^{\prime}\right)=[0: 1: 0]$ and $T_{1}\left(p_{3}^{\prime}\right)=[1: 0: 0]$, and so we can suppose that $p_{1}^{\prime}=[0: 0: 1]$, $p_{2}^{\prime}=[0: 1: 0]$ and $p_{3}^{\prime}=[1: 0: 0]$. Let us consider the configuration in the affine plane $(Z=1)$, with affine coordinates $(x, y) \in \mathbb{C}^{2}$. In this coordinate system, the three lines of $\mathcal{L}^{\prime}$ through $p_{2}^{\prime}$ are of the form $\left(x=a_{1}\right),\left(x=a_{2}\right)$ and $\left(x=a_{3}\right)$, and the three lines through $p_{3}^{\prime}$ are of the form $\left(y=b_{1}\right),\left(y=b_{2}\right)$ and $\left(y=b_{3}\right)$ (see Fig. 2).

Now, each one of the other three lines of the configuration contains $p_{1}=(0,0)$, is neither horizontal nor vertical and contains exactly three points in the set $\left\{\left(a_{i}, b_{j}\right) ; 1 \leqslant i, j \leqslant 3\right\}$. After changing the sub-indexes in the $a_{j^{\prime}}$, if necessary, we can suppose that one of these lines contains the points $\left(a_{j}, b_{j}\right), j=1,2,3$. In this way, one of the other lines contains the points $\left(a_{1}, b_{2}\right)$, $\left(a_{2}, b_{3}\right)$ and $\left(a_{3}, b_{1}\right)$ and the third one the points $\left(a_{1}, b_{3}\right),\left(a_{2}, b_{1}\right)$ and $\left(a_{3}, b_{2}\right)$. This implies that

$$
\frac{b_{1}}{a_{1}}=\frac{b_{2}}{a_{2}}=\frac{b_{3}}{a_{3}}=\alpha, \quad \frac{b_{2}}{a_{1}}=\frac{b_{3}}{a_{2}}=\frac{b_{1}}{a_{3}}=\beta \quad \text { and } \quad \frac{b_{3}}{a_{1}}=\frac{b_{1}}{a_{2}}=\frac{b_{2}}{a_{3}}=\gamma,
$$

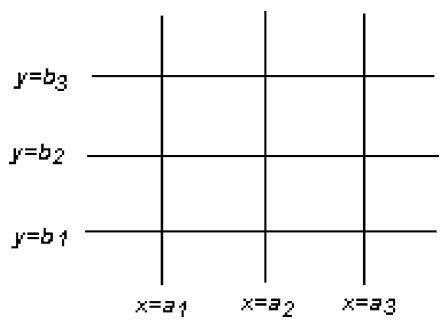

Fig. 2. 
where $\alpha, \beta, \gamma \neq 0$, are distinct complex numbers. It follows from (6) that $\alpha^{3}=\beta^{3}=\gamma^{3}$, and so $\beta / \alpha$ and $\gamma / \alpha$ are distinct primitive cubic roots of the unity. Let us suppose, for instance, that $\beta / \alpha=j$ and $\gamma / \alpha=j^{2}$. This implies that

$$
\left(a_{1}, a_{2}, a_{3}\right)=a_{1}\left(1, j, j^{2}\right) \quad \text { and } \quad\left(b_{1}, b_{2}, b_{3}\right)=\alpha a_{1}\left(1, j, j^{2}\right)
$$

as the reader can check. Now, it is not difficult to see that the automorphism $T$ of $\mathbb{C P}(2)$ defined by

$$
T[X: Y: Z]=\left[a_{1}^{-1} X:\left(\alpha a_{1}\right)^{-1} Y: Z\right]
$$

satisfies (b). Assertions (c) and (d) follow easily from (b). We leave the details for the reader.

\subsection{The family of degree four}

Let $\mathcal{F}$ be a foliation of degree four in $\mathbb{C P}(2)$ for which all the lines of the configuration $\mathcal{C}$ are invariant. In the affine chart $(x, y) \in \mathbb{C}^{2}$ such that the lines of $\mathcal{L}$ are the components of $\left(y^{3}-1\right) \cdot\left(x^{3}-1\right) \cdot\left(y^{3}-x^{3}\right)=0$, such a foliation $\mathcal{F}$ must be given by a differential equation of the form:

$$
\left\{\begin{array}{l}
\frac{d x}{d t}=\left(x^{3}-1\right) \cdot\left[a_{0}+a_{10} x+a_{01} y+b y^{2}\right], \\
\frac{d y}{d t}=\left(y^{3}-1\right) \cdot\left[b_{0}+b_{10} x+b_{01} y+b x^{2}\right]
\end{array}\right.
$$

because the components of $\left(x^{3}-1\right) \cdot\left(y^{3}-1\right)=0$ are invariants by $\mathcal{F}$. We recall that a foliation of degree four in the affine chart $z=(x, y)$, is defined by a differential equation of the form $\frac{d z}{d t}=F(x, y)+g(x, y) R(x, y)$, where $F=(p, q), p$ and $q$ are polynomials of degree $\leqslant 4, g$ is a homogeneous polynomial of degree 4 and $R(x, y)=(x, y)$ (see (2) in $\S 1$ ). Therefore, the homogeneous part of fifth degree in the right side of (7) must be of the form $x^{3} . b y^{2}$ in the first component and $y^{3} \cdot b x^{2}$ in the second. Since the components of $\left(y^{3}-x^{3}\right)=0$ are also invariants, $(0,0)$ must be a singularity of $\mathcal{F}$, so that $a_{0}=b_{0}=0$. On the other hand, the fact that the lines $(y=x),(y=j x)$ and $\left(y=j^{2} x\right)$ are invariant, implies that

$$
\left\{\begin{array}{c}
b_{10}+b_{01}=a_{10}+a_{01} \\
b_{10}+b_{01} j=a_{10} j+a_{01} j^{2} \\
b_{10}+b_{01} j^{2}=a_{10} j^{2}+a_{01} j
\end{array}\right\} \Rightarrow a_{01}=b_{10}=0 \text { and } a_{10}=b_{01}
$$

so that, if we multiply both components of (7) by a convenient constant, we can write (7) in one of the following forms:

$$
\begin{aligned}
& \left\{\begin{array}{l}
\frac{d x}{d t}=P_{\alpha}=\left(x^{3}-1\right) \cdot\left(x-\alpha y^{2}\right), \\
\frac{d y}{d t}=Q_{\alpha}=\left(y^{3}-1\right) \cdot\left(y-\alpha x^{2}\right),
\end{array}\right. \text { or } \\
& \left\{\begin{array}{l}
\frac{d x}{d t}=P_{\infty}=\left(x^{3}-1\right) y^{2}, \\
\frac{d y}{d t}=Q_{\infty}=\left(y^{3}-1\right) x^{2} .
\end{array}\right.
\end{aligned}
$$

We call $\mathcal{F}_{\alpha}^{4}$ the foliation given by (8) and $\mathcal{F}_{\infty}^{4}$ the foliation given by (9). This defines the family $\left(\mathcal{F}_{a}^{4}\right)_{\alpha \in \overline{\mathbb{C}}}$, of degree four. 
Remark 6. - The foliation $\mathcal{F}_{\alpha}^{4}$ is Liouvillian integrable as asserted in Remark 4 of $\S 1$. Let us verify this fact. Observe first that $\mathcal{F}_{\alpha}^{4}$ can be defined by the meromorphic 1 -form

$$
\omega_{\alpha}=\frac{x-\alpha y^{2}}{y^{3}-1} d y-\frac{y-\alpha x^{2}}{x^{3}-1} d x
$$

On the other hand, $d \omega_{\alpha}=\eta \wedge \omega_{\alpha}$ where

$$
\eta=\frac{H+1}{3 H(H-1)} d H, \quad \text { and } \quad H=\frac{y^{3}-1}{x^{3}-1} .
$$

Since $d \eta=0, \mathcal{F}_{\alpha}^{4}$ is Liouvillian integrable.

Remark 7. - Let $S$ be an automorphism of $\mathbb{C P}(2)$ such that $S(\mathcal{C})=\mathcal{C}$ (see (c) and (d) of Proposition 1). It follows from the above arguments, that $S$ sends the family $\left(\mathcal{F}_{a}^{4}\right)_{\alpha \in \overline{\mathbb{C}}}$ in itself, that is, for every $\alpha \in \overline{\mathbb{C}}$, we have that $S^{*}\left(\mathcal{F}_{a}^{4}\right)=\mathcal{F}_{\beta}^{4}$ for some $\beta \in \overline{\mathbb{C}}$.

Proposition 2. - Let $\left(\mathcal{F}_{a}^{4}\right)_{\alpha \in \overline{\mathbb{C}}}$ be as above. Then:

(a) All points of the configuration $\mathcal{C}$ are singularities of $\mathcal{F}_{\alpha}^{4}$, for all $\alpha \in \overline{\mathbb{C}}$.

(b) If $\alpha \notin\left\{1, j, j^{2}, \infty\right\}$, then all singularities of $\mathcal{F}_{\alpha}^{4}$ are non-degenerate. Moreover, these singularities are the following:

(b.1) Twelve singularities of radial type at the points of $\mathcal{P}=\left\{p_{1}, \ldots, p_{12}\right\}$. The foliation $\mathcal{F}_{\alpha}^{4}$ has a local meromorphic first integral of the type $v / u=c t e$, in a neighborhood of each singularity of this set.

(b.2) Nine singularities of saddle type, say $\left\{q_{1}(\alpha), \ldots, q_{9}(\alpha)\right\}$, where, for each $j \in\{1, \ldots, 9\}$, $q_{j}(\alpha) \in \ell_{j}$, a line of $\mathcal{L}$. The foliation $\mathcal{F}_{\alpha}^{4}$ has a local holomorphic first integral of the type $v^{3} \cdot u=$ cte, in a neighborhood of each $q_{j}(\alpha)$.

(c) If $\alpha \in\left\{1, j, j^{2}, \infty\right\}$, then $\operatorname{sing}\left(\mathcal{F}_{\alpha}^{4}\right)=\mathcal{P}$. Among the points in $\mathcal{P}$, nine are of radial type (like in (b.1)) and the three others are contained in one of the subsets $P_{j}, j=1,2,3,4$, as in Proposition 1. Moreover, $\mathcal{F}_{\alpha}^{4}$ has a rational first integral of the type $H_{\alpha}=P_{\alpha} / Q_{\alpha}$, where $P_{\alpha}$ and $Q_{\alpha}$ are the product of three lines in $\mathcal{L}$. These six lines can be chosen in the following way: Suppose that the three non radial singularities of $\mathcal{F}_{\alpha}^{4}$ are $p_{1}, p_{2}, p_{3} \in P_{j}$. Let $\ell_{1}, \ell_{2}, \ell_{3}$ and $\ell_{4}, \ell_{5}, \ell_{6}$ be the lines of $\mathcal{L}$ which pass through two of the points of $P_{j}$. Then $H_{\alpha}=\frac{\ell_{1} \cdot \ell_{2} \cdot \ell_{3}}{\ell_{4} \cdot \ell_{5} \cdot \ell_{6}}$ is a rational first integral of $\mathcal{F}_{\alpha}^{4}$. In particular, for $\alpha=\infty$, the first integral can be chosen to be:

$$
H(x, y):=H_{\infty}(x, y)=\frac{y^{3}-1}{x^{3}-1} .
$$

Proof. - Assertion (a) follows from the fact that the points of $\mathcal{P}$ are the singularities of the algebraic set $\left(Y^{3}-Z^{3}\right) \cdot\left(X^{3}-Z^{3}\right) \cdot\left(Y^{3}-X^{3}\right)=0$, which is invariant for all $\mathcal{F}_{\alpha^{\prime} s}^{4}$. Let us prove assertion (b).

The fact that the singularities of $\mathcal{F}_{\alpha}^{4}$ are non-degenerate for $\alpha \notin\left\{1, j, j^{2}, \infty\right\}$ can be proved by calculating directly its singularities and the Jacobian matrix of the vector field given by (8) in these points. However, another way to prove it, is just observing that $\operatorname{sing}\left(\mathcal{F}_{\alpha}^{4}\right)$ contains exactly $21=4^{2}+4+1$ points, if $\alpha \notin\left\{1, j, j^{2}, \infty\right\}$ (see $\S 1$ ). Let us prove this fact. Consider, for instance the singularities of $\mathcal{F}_{\alpha}^{4}$ in the line $(x=1)$. Since $\mathcal{F}_{\alpha}^{4}$ has degree four, this line must contain five singularities (counted with multiplicities). Four of these singularities are the points $(1,1),(1, j)$, $\left(1, j^{2}\right)$ (in the finite plane) and $[0: 1: 0] \in(Z=0)$. The other one is, of course, the solution of $x-1=y-\alpha x^{2}=0$, which is $(1, \alpha)$. If $\alpha \notin\left\{1, j, j^{2}, \infty\right\}$, then this singularity does not coincide with any of the others, so that, in this case $(x=1)$ contains exactly five singularities of $\mathcal{F}_{\alpha}^{4}$. With 
a similar computation, it can be proved that if $\alpha \notin\left\{1, j, j^{2}, \infty\right\}$, then each line of $\mathcal{L}$ contains exactly five singularities of $\mathcal{F}_{\alpha}^{4}$. This implies that $\mathcal{F}_{\alpha}^{4}$ has nine singularities outside the set $\mathcal{P}$, so that it has exactly 21 singularities. Let us prove (b.1).

Let $Y$ be the polynomial vector field which represents $\mathcal{F}_{\alpha}^{4}$ in an affine chart which contains $p \in \mathcal{P}$. Since $p$ is a non-degenerate singularity of $\mathcal{F}_{\alpha}^{4}$, the Jacobian matrix $D Y(p)$ has two non zero eigenvalues, say $\lambda_{1}, \lambda_{2} \neq 0$. On the other hand, since $\mathcal{F}_{\alpha}^{4}$ has three invariant straight lines through $p$, it is clear that $\lambda_{1}=\lambda_{2}=\lambda$ and $D Y(p)=\lambda$.I, where $I$ is the $2 \times 2$ identity matrix. Now, it follows from the Poincaré's linearization theorem, that $Y$ is linearizable in a neighborhood of $p$ (cf. [2]). Therefore, there exists a (local) holomorphic coordinate system $(U,(u, v))$ such that $p \in U, u(p)=v(p)=0$ and $Y(u, v)=\lambda\left(u \frac{\partial}{\partial u}+v \frac{\partial}{\partial v}\right)$, so that, in these coordinates $v / u$ is a meromorphic first integral of $\mathcal{F}_{\alpha}^{4}$ in $U$.

In order to prove (b.2), let us fix a singularity $q_{j}(\alpha) \in \ell_{j} \in \mathcal{L}$, say $(1, \alpha) \in \ell_{1}=(x=1)$. Consider the vector field $X$ which represents $\mathcal{F}_{\alpha}^{4}$ in the affine chart $(x, y)$ (see (8)):

$$
X(x, y)=\left[\left(x^{3}-1\right) \cdot\left(x-\alpha y^{2}\right)\right] \frac{\partial}{\partial x}+\left[\left(y^{3}-1\right) \cdot\left(y-\alpha x^{2}\right)\right] \frac{\partial}{\partial y} .
$$

A direct computation shows that

$$
D X(1, \alpha)=\left(\begin{array}{cc}
3\left(1-\alpha^{3}\right) & 0 \\
0 & \alpha^{3}-1
\end{array}\right)
$$

and so the eigenvalues of $D X(1, \alpha)$ are $\lambda_{1}=3\left(1-\alpha^{3}\right)$ and $\lambda_{2}=\alpha^{3}-1$ and satisfy $\lambda_{1}=-3 . \lambda_{2}$, where $\lambda_{1}$ is the eigenvalue in the normal direction to $\ell_{1}$ and $\lambda_{2}$ in the tangent direction to $\ell_{1}$. With a similar calculation (or using Proposition 1), it can be proved that this property is true for all singularities $q_{j}(\alpha), j=2, \ldots, 9$. It remains to prove that $\mathcal{F}_{\alpha}^{4}$ has a local holomorphic first integral in a neighborhood of $q_{j}(\alpha)$ of the form $v^{3} \cdot u=c t e$. In order to prove this fact, we recall that the other four singularities of $\mathcal{F}_{\alpha}^{4}$ on $\ell_{j}$ are radial and we will consider the resolution of these singularities by blowing-up. From now on, we shall use the following notations:

(i) $M$ will be the manifold obtained from $\mathbb{C P}(2)$ by blowing-up in the twelve points of $\mathcal{P}$. The blowing-up map will be denoted by $\Pi: M \rightarrow \mathbb{C P}(2)$.

(ii) $\tilde{\mathcal{F}}_{\alpha}$ will be the strict transform of $\mathcal{F}_{\alpha}^{4}$ by $\Pi$.

(iii) $\tilde{\ell}_{j}, j=1, \ldots, 9$, will be the strict transform of the line $\ell_{j} \in \mathcal{L}$ by $\Pi$ and $\mathcal{L}=\bigcup_{j=1}^{9} \tilde{\ell}_{j}$.

(iv) $D_{i}$ will be the divisor $\Pi^{-1}\left(p_{i}\right)$.

Observe that $\tilde{\mathcal{F}}_{\alpha}$ has just one singularity on $\tilde{\ell}_{j}$, say $\tilde{q}_{j}(\alpha)$, where $\Pi\left(\tilde{q}_{j}(\alpha)\right)=q_{j}(\alpha)$. This implies that $\tilde{\ell}_{j} \backslash\left\{\tilde{q}_{j}(\alpha)\right\}$ is a leaf of $\tilde{\mathcal{F}}_{\alpha}$ biholomorphic to $\mathbb{C}$. Therefore, the holonomy of this leaf is trivial, and this implies that the holonomy of the local separatrix of $\tilde{q}_{j}(\alpha)$ contained in $\tilde{\ell}_{j}$ is the identity. In particular, this holonomy is linearizable. It follows from a Theorem of Mattei and Moussu (cf. [18]), that $\tilde{\mathcal{F}}_{\alpha}$ is linearizable in a neighborhood of $\tilde{q}_{j}(\alpha)$. Since $\Pi$ is a biholomorphism in a neighborhood of $\tilde{q}_{j}(\alpha)$, the same is true for $\mathcal{F}_{\alpha}^{4}$ in a neighborhood of $q_{j}(\alpha)$. Therefore, we can suppose that in a suitable holomorphic coordinate system $(V,(u, v))$ around $q_{j}(\alpha)$, we have

$$
f(u, v) \cdot X(u, v)=3 u \frac{\partial}{\partial u}-v \frac{\partial}{\partial v},
$$

where $f \in \mathcal{O}^{*}(V)$ and $V \cap \ell_{j}=(u=0)$. This implies that $v^{3}$. $u$ is a holomorphic first integral of $\mathcal{F}_{\alpha}^{4}$ in $V$, which proves (b.2). 
Let us consider now the rational map $H(x, y)=\frac{y^{3}-1}{x^{3}-1}=\frac{P}{Q}$. Then

$$
\frac{d H}{H}=\frac{d P}{P}-\frac{d Q}{Q}=\frac{3 y^{2} d y}{y^{3}-1}-\frac{3 x^{2} d x}{x^{3}-1}=\frac{3}{\left(x^{3}-1\right)\left(y^{3}-1\right)} \cdot\left[\left(x^{3}-1\right) y^{2} d y-\left(y^{3}-1\right) x^{2} d x\right] .
$$

This implies that $H=H_{\infty}$ is a rational first integral for $\mathcal{F}_{\infty}^{4}$ (see (9)). Observe that $H-1=$ $\frac{y^{3}-x^{3}}{x^{3}-1}$ and $\frac{1}{H}-1=\frac{x^{3}-y^{3}}{y^{3}-1}$ are also rational first integrals for $\mathcal{F}_{\infty}^{4}$. Therefore, if we choose six lines of $\mathcal{L}$, say $\ell_{1}, \ldots, \ell_{6}$, such that $\ell_{1}, \ldots, \ell_{3}$ pass through one of the points of $P_{1}=\{[1: 0: 0]$, $[0: 1: 0],[0: 0: 1]\}$ and $\ell_{4}, \ldots, \ell_{6}$ pass through another one, then $\frac{\ell_{1} \cdot \ell_{2} \cdot \ell_{3}}{\ell_{4} \cdot \ell_{5} \ell_{6}}$ is a rational first integral for $\mathcal{F}_{\infty}^{4}$. Now let $P_{j}, j \in\{2,3,4\}$, be another subset of $\mathcal{P}$ as in Proposition 1. It follows from (c) of Proposition 1 that there exists an automorphism $S$ of $\mathbb{C P}(2)$ such that $S(\mathcal{C})=\mathcal{C}$ and $S\left(P_{j}\right)=P_{1}$. Since $S(\mathcal{C})=\mathcal{C}$ we must have $S^{*}\left(\mathcal{F}_{\infty}^{4}\right)=\mathcal{F}_{T}^{4}$ for some $T \in \overline{\mathbb{C}} \backslash\{\infty\}$. In fact, $T \in\left\{1, j, j^{2}\right\}$, because for $\alpha \notin\left\{1, j, j^{2}, \infty\right\}, \mathcal{F}_{\alpha}^{4}$ has 21 singularities, whereas $\mathcal{F}_{\infty}^{4}$ has 12 singularities. It follows that $H \circ S$ is a rational first integral of $\mathcal{F}_{T}$ of the type required in (c). This finishes the proof of Proposition 2.

One of the main facts about the family $\left(\mathcal{F}_{\alpha}^{4}\right)_{\alpha \in \overline{\mathbb{C}}}$, is the following:

Proposition 3. - If $\alpha \neq \beta$ then $\tilde{\mathcal{F}}_{\alpha}$ and $\tilde{\mathcal{F}}_{\beta}$ are transversal outside $\mathcal{L}=\tilde{\ell}_{1} \cup \cdots \cup \tilde{\ell}_{9}$.

Proof. - We will prove the proposition for $\alpha, \beta \neq \infty$ and leave for the reader the case where either $\alpha=\infty$ or $\beta=\infty$. Let us prove first that $\tilde{\mathcal{F}}_{\alpha}$ and $\tilde{\mathcal{F}}_{\beta}$ are transversal outside $\mathcal{L} \cup D_{1} \cup \cdots \cup D_{12}$. Since $\Pi$ is a biholomorphism outside $D_{1} \cup \cdots \cup D_{12}$, it is sufficient to prove that $\mathcal{F}_{\alpha}^{4}$ and $\mathcal{F}_{\beta}^{4}$ are transversal outside $\ell_{1} \cup \cdots \cup \ell_{9}$. By considering the determinant of the components of vector fields which define $\mathcal{F}_{\alpha}^{4}$ and $\mathcal{F}_{\beta}^{4}$ (see (8)), we get:

$$
P_{\alpha} \cdot Q_{\beta}-P_{\beta} \cdot Q_{\alpha}=(\beta-\alpha) \cdot\left(x^{3}-1\right) \cdot\left(y^{3}-1\right) \cdot\left(y^{3}-x^{3}\right) .
$$

This proves that $\mathcal{F}_{\alpha}^{4}$ and $\mathcal{F}_{\beta}^{4}$ are transversal outside $\ell_{1} \cup \cdots \cup \ell_{9} \cup(Z=0)$. In order to verify that they are tranversal in $(Z=0) \backslash\left(\ell_{1} \cup \cdots \cup \ell_{9}\right)=(Z=0) \backslash\{[1: 0: 0],[0: 1: 0]\}$ we can use (c) of Proposition 1. It follows from (c) of Proposition 1, that it is possible to find an automorphism $S$ of $\mathbb{C P}(2)$ such that $S(\mathcal{C})=\mathcal{C}$ and $S((Z=0) \backslash\{[1: 0: 0],[0: 1: 0]\}) \subset \mathbb{C}^{2}$. It follows from Remark 7 and the above computation that $\mathcal{F}_{\alpha}^{4}$ and $\mathcal{F}_{\beta}^{4}$ are transversal in $(Z=0) \backslash\{[1: 0: 0]$, $[0: 1: 0]\}$. This proves that $\tilde{\mathcal{F}}_{\alpha}$ and $\tilde{\mathcal{F}}_{\beta}$ are transversal outside $\mathcal{L} \cup D_{1} \cup \cdots \cup D_{12}$.

It remains to prove that $\tilde{\mathcal{F}}_{\alpha}$ and $\tilde{\mathcal{F}}_{\beta}$ are transversal in $\left(D_{1} \cup \cdots \cup D_{12}\right) \backslash \mathcal{L}$. Observe that, (d) of Proposition 1 and Remark 7, imply that it is sufficient to prove that they are tranversal in $D_{j} \backslash \mathcal{L}$, for some $j \in\{1, \ldots, 12\}$. Since $p_{1}=(0,0) \in \mathcal{P}$, we will prove that they are tranversal in $D_{1} \backslash \mathcal{L}$, by doing a blowing-up $\pi$ at $0 \in \mathbb{C}^{2}$. Consider, for instance this blowing-up in the chart $(u, x) \in \mathbb{C}^{2}$, where $\pi(u, x)=(x, u . x)$. A straightforward computation shows that the strict transform of $\tilde{\mathcal{F}}_{\alpha}$ can be defined in this chart by:

$$
\left\{\begin{array}{l}
\frac{d u}{d t}=\alpha\left(u^{3}-1\right)+x \cdot h_{1}(u, x), \\
\frac{d x}{d t}=1+x \cdot h_{2}(u, x)
\end{array}\right.
$$

where $h_{1}$ and $h_{2}$ are polynomials. In this chart, the equation of the divisor $D_{1}$ is $(x=0)$, so that the slopes of $\tilde{\mathcal{F}}_{\alpha}$ and $\tilde{\mathcal{F}}_{\beta}$ along $D_{1}$ are given by $\frac{d u}{d x}=\alpha\left(u^{3}-1\right)$ and $\frac{d u}{d x}=\beta\left(u^{3}-1\right)$, respectively. This implies that they are tranversal along $D_{1}$, outside $\left(u^{3}-1=0\right)$, which is the equation of $\mathcal{L}$ in this chart. With a similar computation, it is possible to prove that they are also tranversal outside $\mathcal{L}$ in the other chart $(v, y)$, where $\pi(v, y)=(v . y, y)$. We leave this computation for the reader. This finishes the proof of Proposition 3. 


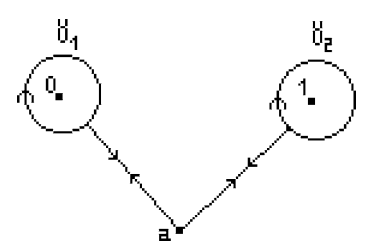

Fig. 3.

Now, consider the first integral $H=\frac{y^{3}-1}{x^{3}-1}$ of $\mathcal{F}_{\infty}^{4}$ and set $h=H \circ \Pi$. Since the base points of $H$ are contained in $\mathcal{P}$ and these base points are radial points for $\mathcal{F}_{\infty}$, it is not difficult to see that $h: M \rightarrow \overline{\mathbb{C}}$ is holomorphic and the singular fibers of $h$ are $h^{-1}(0), h^{-1}(1)$ and $h^{-1}(\infty)$. Moreover, if $V=M \backslash h^{-1}\{0,1, \infty\}$ and $W=\overline{\mathbb{C}} \backslash\{0,1, \infty\}$, then $\left.h\right|_{V}: V \rightarrow W$ is an elliptic regular fibration. The idea of the proof of the main Theorem is to use Proposition 3, this fibration and the theory of foliations transversal to the fibers of a fibration (see for instance [12] or [6]). According to this theory, if we fix one of the foliations $\tilde{\mathcal{F}}_{\alpha}, \alpha \neq \infty$ and a fiber of $\left.h\right|_{V}$, say $T_{a}=h^{-1}(a)$, then there exists a group representation

$$
\mathcal{H}_{\alpha}: \Pi_{1}(W, a) \rightarrow \operatorname{diff}\left(T_{a}\right),
$$

called global holonomy representation, with the following properties:

(I) If $L_{o}$ is a leaf of $\left.\tilde{\mathcal{F}}_{\alpha}\right|_{V}$, then $\left.h\right|_{L_{o}}: L_{o} \rightarrow W$ is a covering map.

(II) Given a closed path $\gamma:[0,1] \rightarrow W$ such that $\gamma(0)=\gamma(1)=a$, with class $[\gamma] \in \Pi_{1}(W, a)$, and a point $q \in L_{o} \cap T_{a}$, then $\mathcal{H}_{\alpha}([\gamma])(q)$ is the end point of the unique path $\tilde{\gamma}:[0,1] \rightarrow L_{o}$ such that $\tilde{\gamma}(0)=q$ and $h \circ \tilde{\gamma}=\gamma$. In particular, the orbit of $q$ by $\mathcal{H}_{\alpha}([\gamma])$ is contained in $L_{o} \cap T_{a}$.

(III) If we denote by $G_{\alpha}$ the subgroup $\mathcal{H}_{\alpha}\left(\Pi_{1}(W, a)\right)$ of $\operatorname{diff}\left(T_{a}\right)$, then

$$
L_{o} \cap T_{a}=\left\{f(q): f \in G_{\alpha}\right\}
$$

that is $L_{o} \cap T_{a}$ is the orbit of some point $q \in L_{o} \cap T_{a}$ by the group of diffeomorphims $G_{\alpha}$.

Remark 8. - Since the foliation $\tilde{\mathcal{F}}_{\alpha}$ and the fibration $\left.h\right|_{V}$ are holomorphic, it follows that all elements $f \in G_{\alpha}$ are automorphims of the elliptic curve $T_{a}$. Moreover, if we fix $\gamma \in \Pi_{1}(W, a)$ then we can define a map $F_{\gamma}: \mathbb{C} \times T_{a} \rightarrow T_{a}$ by

$$
F_{\gamma}(\alpha, q)=\mathcal{H}_{\alpha}(\gamma)(q)
$$

where $\mathcal{H}_{\alpha}$ is the global holonomy of $\tilde{\mathcal{F}}_{\alpha}$. It follows from the theorem of holomorphic dependence of the solutions of a holomorphic differential equation with respect to parameters and initial conditions, that the map $F_{\gamma}$ is holomorphic in both variables.

On the other hand, $W=\overline{\mathbb{C}} \backslash\{0,1, \infty\}=\mathbb{C} \backslash\{0,1\}$, so that $\Pi_{1}(W, a)$ is generated by the two closed paths $\gamma_{1}$ and $\gamma_{2}$ illustrated in Fig. 3. We will denote by $f_{\alpha}$ and $g_{\alpha}$ the automorphisms of $T_{a}$ defined by

$$
f_{\alpha}(q)=F_{\gamma_{1}}(\alpha, q) \quad \text { and } \quad g_{\alpha}(q)=F_{\gamma_{2}}(\alpha, q),
$$

respectively. In particular, $G_{\alpha}$ is generated by $f_{\alpha}$ and $g_{\alpha}$. In the next result, we will see how $f_{\alpha}$ and $g_{\alpha}$ look like.

Proposition 4. - Let $h, T_{a}=h^{-1}(a), \tilde{\mathcal{F}}_{\alpha}, f_{\alpha}$ and $g_{\alpha}$ be as before. Then:

(a) For all $b \in W$, the fiber $T_{b}=h^{-1}(b)$ is biholomorphic to the torus $\mathbb{C} / \Gamma$, where $\Gamma=\langle 1, j\rangle$ is lattice generated by 1 and $j=\mathrm{e}^{2 \pi \mathrm{i} / 3}$. 
(b) There exists a holomorphic map $A: \mathbb{C} \rightarrow \mathbb{C} / \Gamma$, such that for every $\alpha \in \mathbb{C}$, the global holonomy group $G_{\alpha}$ of $\tilde{\mathcal{F}}_{\alpha}$ in the fiber $T_{a}$, written in a suitable holomorphic universal covering $P_{\alpha}: \mathbb{C} \rightarrow T_{a}$, is generated by $f_{\alpha}(z)=j . z$ and $g_{\alpha}(z)=j . z+A(\alpha)$.

(c) The map $A: \mathbb{C} \rightarrow \mathbb{C} / \Gamma$ is not constant.

Proof. - Since the fibers of $\left.h\right|_{V}$ are tranverse to $\tilde{\mathcal{F}}_{\alpha}, \alpha \neq \infty$, it follows that all these fibers are biholomorphic. The fact that they are biholomorphic to $\mathbb{C} / \Gamma$ is a consequence of the following:

AsSERTION. $-f_{\alpha}^{3}=i d$ (resp. $g_{\alpha}^{3}=i d$ ), $f_{a}$ (resp. $g_{\alpha}$ ) has a fixed point, but $f_{\alpha} \neq i d$ (resp. $\left.g_{\alpha} \neq i d\right)$.

It is well-known that an elliptic curve which admits an automorphism satisfying the properties of $f_{\alpha}$ in the assertion is biholomorphic to $\mathbb{C} / \Gamma$ (cf. [1]).

Proof of the assertion. - We will use (b.2) of Proposition 2. Consider the singular level $h^{-1}(0)=D_{1} \cup \tilde{\ell}_{1} \cup \tilde{\ell}_{2} \cup \tilde{\ell}_{3}$, where $D_{1}$ is the divisor $\Pi^{-1}(0)$ and $\tilde{\ell}_{1}, \tilde{\ell}_{2}$ and $\tilde{\ell}_{3}$ are the strict tranforms of $(y=x),(y=j x)$ and $\left(y=j^{2} x\right)$, respectively. Let $\tilde{q}_{k}(\alpha) \in \tilde{\ell}_{k}$ be the singularity of saddle type of $\tilde{\mathcal{F}}_{\alpha}$, such that $\Pi\left(\tilde{q}_{k}(\alpha)\right)=q_{k}(\alpha)=\left(j^{k-1} / \alpha, j^{2(k-1)} / \alpha\right) \in\left(y=j^{k-1} x\right)$, $k=1,2,3$. Since $\Pi$ is a biholomorphism outside $\bigcup_{i=1}^{12} D_{i}$, there are neighborhoods $\widetilde{U}_{k}$ of $\tilde{q}_{k}(\alpha)$ and $U_{k}$ of $q_{k}(\alpha)$ such that $\left.\Pi\right|_{\widetilde{U}_{k}}: \widetilde{U}_{k} \rightarrow U_{k}$ is a biholomorphism. In particular, the foliations $\tilde{\mathcal{F}}_{\alpha}$ and $\mathcal{F}_{\alpha}^{4}$ are analytically equivalent in $\widetilde{U}_{k}$ and $U_{k}$, respectively. For simplicity, let us consider the case $k=1$. In this case, there exists an analytic coordinate system $(u, v): \widetilde{U}_{1} \rightarrow \mathbb{C}^{2}$ such that:

(i) $u\left(\tilde{q}_{1}(\alpha)\right)=v\left(\tilde{q}_{1}(\alpha)\right)=0$ and $\tilde{\ell}_{1} \cap \widetilde{U}_{1}=(u=0)$.

(ii) $\left.\tilde{\mathcal{F}}_{\alpha}\right|_{\widetilde{U}_{1}}$ is defined in $\widetilde{U}_{1}$ by the differential equation $3 u d v+v d u=0$, so that $v^{3} \cdot u$ is a holomorphic first integral of $\left.\tilde{\mathcal{F}}_{\alpha}\right|_{\widetilde{U}_{1}}$.

(iii) The maps $\left.h\right|_{\widetilde{U}_{1}}=\left.H \circ \Pi\right|_{\widetilde{U}_{1}}$ and $\left.H\right|_{U_{1}}$ are equivalent.

It follows from (ii) that the holonomy of the local separatrix $(v=0)$ of $\tilde{q_{1}}(\alpha)$ in a transversal $\left(u=u_{o}\right)\left(u_{o} \neq 0\right)$, is $f_{o}(v)=\mathrm{e}^{-2 \pi \mathrm{i} / 3} \cdot v=j^{2} \cdot v$. In particular, we have $f_{o} \neq i d$ and $f_{o}^{3}=i d$. This implies that, if $\Sigma \subset \widetilde{U}_{1}$ is any transversal to $(v=0)$ through a point $q_{o} \in(v=0)$ and $f:\left(\Sigma, q_{o}\right) \rightarrow\left(\Sigma, q_{o}\right)$ is the holonomy of $(v=0)$ in this transversal, then

(iv) $f\left(q_{o}\right)=q_{o}, f \neq i d$ and $f^{3}=i d$.

Now, observe that $\left.H\right|_{U_{1}}=1+\frac{y^{3}-x^{3}}{x^{3}-1}$, and so $h$ has multiplicity one along the curve $\tilde{\ell}_{1}$, that is, there exists an analytic coordinate system $\left(W_{1},\left(u_{1}, v_{1}\right)\right)$ around $(0,0)$ such that

(v) $W_{1} \subset \widetilde{U}_{1}, W_{1}=\left\{\left(u_{1}, v_{1}\right) ;\left|u_{1}\right|,\left|v_{1}\right|<\delta\right\},(u=0) \cap W_{1}=\left(u_{1}=0\right), v_{1}=v$ and $h\left(u_{1}, v_{1}\right)=1+u_{1}$.

Let us suppose for a moment that $|a|<\delta$ and that the curve $\gamma_{1}$ is contained in $D_{\delta}=$ $\{z ;|z-1|<\delta\}$. In this case, the fiber $T_{a}=h^{-1}(a)$ cuts $W_{1}$ in the transversal to $\left(v_{1}=0\right)$, $\Sigma=\left\{\left(u_{1}, v_{1}\right) ; u_{1}=1+a\right\}$. Moreover, the global holonomy $f_{\alpha}$ of $\gamma_{1}$, is such that $\left.f_{\alpha}\right|_{\Sigma}=f^{-1}$, where $f$ is the holonomy of the local separatrix $\left(v_{1}=0\right)$ as in (iv) (observe that $\gamma_{1}$ in Fig. 3 is oriented in the negative sense of $\mathbb{C}$ ). Since $f_{\alpha}$ is holomorphic, (iv) implies that $f_{\alpha} \neq i d$ and $f_{\alpha}^{3}=i d$. The proof of the assertion in the general case, that is $\gamma_{1} \not \subset D_{\delta}$, follows from this case. In fact, it is enough to observe that $f_{\alpha}=k^{-1} \circ f_{1} \circ k$, where $f_{1}$ is the global holonomy of simple curve $\gamma(s)=1+\frac{\delta}{2} \exp (2 \pi \mathrm{i} s), s \in[0,1]$, contained in $D_{\delta}$ and $k: h^{-1}(a) \rightarrow h^{-1}\left(\frac{1+\delta}{2}\right)$ is the global holonomy of a simple curve joining $a$ to $1+\frac{\delta}{2}$ (see Fig. 3). This proves the assertion.

Remark 9. - It follows from Remark 8 that the assertion is also true for $\alpha \in\left\{1, j, j^{2}\right\}$.

Let us fix a holomorphic universal covering $P: \mathbb{C} \rightarrow T_{a}$, with associated lattice $\Gamma$. It follows from the assertion, that the liftings of $f_{\alpha}$ and $g_{\alpha}$, which we will denote by the same symbols, can 
be written as

$$
f_{\alpha}(z)=j . z+a(\alpha) \quad \text { and } \quad g_{\alpha}(z)=j . z+b(\alpha),
$$

where $a: \mathbb{C} \rightarrow \mathbb{C} / \Gamma$ and $b: \mathbb{C} \rightarrow \mathbb{C} / \Gamma$ are holomorphic (observe that the senses of $\gamma_{1}$ and $\gamma_{2}$ were chosen in Fig. 3 in such a way that $\left.f_{\alpha}^{\prime}=g_{\alpha}^{\prime}=j\right)$. On the other hand, the point $z_{o}(\alpha)=\frac{a(\alpha)}{1-j}$ is a fixed point of $f_{\alpha}$, so that $k_{\alpha}^{-1} \circ f_{\alpha} \circ k_{\alpha}(z)=j . z$, where $k_{\alpha}(z)=z+z_{o}(\alpha)$. Since $k_{\alpha}^{-1} \circ g_{\alpha} \circ k_{\alpha}(z)=j . z+A(\alpha)$, where $A(\alpha)=b(\alpha)-a(\alpha)$, we obtain that in the covering $P_{\alpha}=P \circ k_{\alpha}: \mathbb{C} \rightarrow T_{a}$ the generators $f_{\alpha}$ and $g_{\alpha}$ of the global holonomy, are written as in (b). This proves (b).

In order to prove (c), it is enough to exhibit two foliations $\tilde{\mathcal{F}}_{\alpha_{1}}$ and $\tilde{\mathcal{F}}_{\alpha_{2}}$, such that the global holonomy groups, $G_{\alpha_{1}}$ and $G_{\alpha_{2}}$ are finite and have a different number of elements. In fact, we will prove that, in the representation of (b), $G_{1}$ has three elements (in this case $G_{1}=\langle j . z\rangle$ and $\left.f_{1}(z)=g_{1}(z)=j . z\right)$ and $G_{0}$ has at least nine elements, so that $A(1)=0(\bmod \Gamma)$ and $A(0) \neq 0$ $(\bmod \Gamma)$.

(c.1) We assert that $G_{1}$ has three elements.

In fact, as we have seen in (c) of Proposition $2, \mathcal{F}_{1}^{4}$ has a rational first integral, say $H_{1}=\frac{p_{1}}{q_{1}}$, where $p_{1}$ and $q_{1}$ are the product of three lines which pass through two of the points of $P_{2}=\left\{(1,1),\left(j, j^{2}\right),\left(j^{2}, j\right)\right\}$, so that we can take

$$
H_{1}(x, y)=\frac{\left(x-j^{2}\right)(y-j)\left(y-j^{2} x\right)}{(x-j)\left(y-j^{2}\right)(y-j x)} .
$$

It follows from Bézout's Theorem, that the general fiber $H_{1}^{-1}(c)$ of $H_{1}$, cuts $H_{\infty}^{-1}(a)=\Pi\left(T_{a}\right)$ in $3 \times 3=9$ points, counted with multiplicities. Now, observe that, between the twelve points of $\mathcal{P}, H_{1}^{-1}(c)$ does not pass through the points of $P_{2}$ and $H_{\infty}^{-1}(a)$ does not pass through the points of $P_{1}=\{(0,0),[1: 0: 0],[0: 1: 0]\}$. It follows that $\mathcal{P} \cap H_{1}^{-1}(c) \cap H_{\infty}^{-1}(a)$ contains six points of $\mathcal{P}$. As the reader can check, the intersection number of $H_{1}^{-1}(c)$ and $H_{\infty}^{-1}(a)$ in each of these points is one. This implies that, the general leaf of $\tilde{\mathcal{F}}_{1}$ cuts the fiber $h^{-1}(a)=T_{a}$ in $3=9-6$ points, so that $\# G_{1}=3$. Therefore $f_{1}(z)=g_{1}(z)=j . z$ and $G_{1}=\langle j . z\rangle$.

(c.2) We assert that $G_{0}$ has at least nine elements, so that $G_{0} \neq G_{1}$ and $A(0) \neq 0(\bmod \Gamma)$.

First of all observe that

$$
H_{0}(x, y)=\frac{p_{0}}{q_{0}}=\frac{x^{3}\left(y^{3}-1\right)}{y^{3}\left(x^{3}-1\right)}
$$

is a rational first integral of $\mathcal{F}_{0}^{4}$. In fact, we have

$$
q_{0}^{2} \cdot d H_{0}=q_{0} d p_{0}-p_{0} d q_{0}=3 x^{2} y^{2}\left[x\left(x^{3}-1\right) d y-y\left(y^{3}-1\right) d x\right]
$$

which implies that $H_{0}$ is a first integral of $\mathcal{F}_{0}^{4}$ (see (8)).

Now, the line $\ell=(x=0)$, which is contained in $H_{0}^{-1}(0)$, cuts the general level $H^{-1}(a)$ in three points, $\left(0, y_{k}\right)$, where $y_{1}, y_{2}, y_{3}$ are cubic roots of $1-a$. Since $H_{0}$ has multiplicity three along $(x=0)$, it follows that, if $|b|$ is small, then $H_{0}^{-1}(b)$ cuts $H^{-1}(a)$ in at least nine points, which are near the points $\left(0, y_{k}\right), k=1,2,3$. But $\left(0, y_{k}\right) \notin \mathcal{P}, k=1,2,3$, so that if $|b|$ is small, none of these nine points is in $\mathcal{P}$. This implies that $h^{-1}(a)$ contains at least nine points of the generic leaf of $\tilde{\mathcal{F}}_{0}$. Therefore, $\#\left(G_{0}\right) \geqslant 9$, which proves (c.2) and the proposition.

In the next result, we will see how a group $G$ of the form $\langle j . z, j . z+A\rangle$ looks like. We will also characterize the finite groups of this form. 
PROPOSITION 5. - Let $G$ be a subgroup of the group of automorphisms of $\mathbb{C} / \Gamma$, generated by two elements $f(z)=j$. $z$ and $g(z)=j . z+$ A. Then

$$
G=\left\{k ; k(z)=c . z+d . A \text { where } c \in\left\{1, j, j^{2}\right\} \text { and } d \in \mathbb{Z} \oplus j . \mathbb{Z}\right\} .
$$

In particular, the following assertions are equivalent:

(a) $G$ is finite.

(b) There exists $n \in \mathbb{Z}$ such that $n A \in \Gamma$.

(c) G has a finite orbit in $\mathbb{C} / \Gamma$.

Proof. - We will prove (14) and leave the proof of the equivalence between (a), (b) and (c) for the reader. Set

$$
G_{1}=\left\{k ; k(z)=c . z+d . A \text { where } c \in\left\{1, j, j^{2}\right\} \text { and } d \in \mathbb{Z} \oplus j \cdot \mathbb{Z}\right\}
$$

It is not difficult to see that $G_{1}$ is a subgroup of $A u t(\mathbb{C} / \Gamma)$. Since $f, g \in G_{1}$, it is clear that $G \subset G_{1}$.

On the other hand, since $f$ and $g$ have order three and $G=\langle f, g\rangle$, any $k \in G$ can be written as a word

$$
k=f^{\alpha_{1}} \circ g^{\beta_{1}} \circ \cdots \circ f^{\alpha_{r}} \circ g^{\beta_{r}},
$$

where $r \geqslant 1,0 \leqslant \alpha_{1}, \beta_{r} \leqslant 2,1 \leqslant \alpha_{m}, \beta_{n} \leqslant 2$, if $2 \leqslant m \leqslant r$ and $1 \leqslant n \leqslant r-1$. Let us define the length of the word $k$ as $|k|:=\alpha_{1}+\cdots+\alpha_{r}+\beta_{1}+\cdots+\beta_{r}$ and prove that any $k \in G_{1}$ is in $G$, by induction on $|k|$. If $|k|=0$ then $k$ is the identity map, so that $k \in G_{1}$. Suppose that for any element $k \in G$ with $1 \leqslant|k|<n$ we have $k \in G_{1}$. Let $h \in G$ be of length $n$. Then $h=f \circ k$ or $h=g \circ k$, where $|k|<n$. By the induction hypothesis, we have $k(z)=c . z+d . A$, where $c \in\left\{1, j, j^{2}\right\}$ and $d \in \mathbb{Z} \oplus j . \mathbb{Z}$. On the other hand, $f \circ k(z)=j . c . z+j . d . A$ and $g \circ k(z)=$ j.c. $z+(j . d+1) \cdot A$, so that $h \in G_{1}$, as the reader can check. This finishes the proof of the proposition.

As a consequence, we obtain the following:

COROLLARY. - The following assertions are equivalent:

(a) $\mathcal{F}_{\alpha}^{4}$ has a rational first integral.

(b) There exists $n \in \mathbb{Z}$ such that $n A(\alpha) \in \Gamma$, where $A(\alpha)$ is like in Proposition 4.

(c) $\mathcal{F}_{\alpha}^{4}$ has an algebraic leaf which is not contained in the lines of $\mathcal{L}$.

Proof. - Clearly (a) $\Rightarrow$ (c). Let us prove that (c) $\Rightarrow$ (b) $\Rightarrow$ (a).

(c) $\Rightarrow$ (b). Let $L$ be an algebraic leaf of $\mathcal{F}_{\alpha}^{4}$ which is not contained in the lines of $\mathcal{L}$ and $\tilde{L}$ be its strict transform by $\Pi$. Since $L$ is algebraic, it follows that $\tilde{L} \cap T_{a}$ is finite. But, $\tilde{L} \cap T_{a}$ is an orbit of $G_{\alpha}$, so that $G_{\alpha}$ has a finite orbit. Therefore (c) $\Rightarrow$ (b).

(b) $\Rightarrow$ (a). Observe that (b) implies that $G_{\alpha}$ is finite, say $\# G_{\alpha}=m$. Therefore, if $L \not \subset \mathcal{L}$ is a leaf of $\tilde{\mathcal{F}}_{\alpha}$, then $\# L \cap T_{b} \leqslant m$, for any fiber $T_{b}=h^{-1}(b), b \in W$. On the other hand, if $L$ does not contain the local separatrices of the singularities $q_{j}(\alpha)$, then $L$ cuts the singular levels $h^{-1}(c), c=0,1, \infty$, transversely in the divisors contained in these levels (Proposition 3), say $D_{1}, D_{2}$ and $D_{3}$. It is not difficult to see that this implies that $\# L \cap D_{j} \leqslant m$ and that $L$ can be covered by a finite number of relatively compact disks, so that $L$ is compact. In particular, $\mathcal{F}_{\alpha}^{4}$ has infinitely many algebraic solutions and so a meromorphic first integral, by Darboux's Theorem. 
We have already proved assertion (a) of the main Theorem for the family $\left(\mathcal{F}_{\alpha}^{4}\right)_{\alpha \in \overline{\mathbb{C}}}$. In order to prove (b) we have to define the set $E$ of the statement of the main Theorem. Let

$$
E=\{\alpha \in \mathbb{C} ; \text { there exists } n \in \mathbb{Z} \text { such that } n . A(\alpha) \in \Gamma\} \cup\{\infty\} .
$$

Since $A: \mathbb{C} \rightarrow \mathbb{C}(\bmod \Gamma)$ is holomorphic and not constant, it follows that $E$ is countable and dense. The Corollary of Proposition 5 implies that $\mathcal{F}_{\alpha}^{4}$ has a rational first integral $F_{\alpha}=\frac{P_{\alpha}}{Q_{\alpha}}$ if, and only if, $\alpha \in E$. Let $d(\alpha)$ be the degree of a generic level curve of $F_{\alpha}$, say $L_{c}:=\left(P_{\alpha}-c \cdot Q_{\alpha}=0\right)$, and $\tilde{L}_{c}$ be the strict transform of $L_{c}$ by $\Pi$. By Bézout's Theorem, we have

$$
3 . d(\alpha)=\#\left(L_{c} \cap H_{\infty}^{-1}(a)\right) \geqslant \#\left(\tilde{L}_{c} \cap h^{-1}(a)\right)=\# G_{\alpha},
$$

and so $d(\alpha) \geqslant \frac{1}{3} \# G_{\alpha}$. On the other hand, given $A \in \mathbb{C}^{*}$ such that there exists $n \in \mathbb{Z}$ with n. $A \in \Gamma$, let

$$
k=k(A)=\min \{n \in \mathbb{Z} ; n>0 \text { and } n . A \in \Gamma\} .
$$

Then, it is not difficult to see from (14) that $\#(\langle j . z, j . z+A\rangle) \geqslant 3 . k^{2}$. Therefore, if $\alpha \in E$ then $d(\alpha) \geqslant k(A(\alpha))^{2}$. Given $k_{o} \in \mathbb{N}$, let

$$
E_{k_{o}}=\left\{\alpha \in E ; k(A(\alpha)) \leqslant k_{o}\right\} \cup\{\infty\} .
$$

It is not difficult to see that $E_{k_{o}}$ is finite and that, if $\alpha \notin E_{k_{o}}$, then $d(\alpha) \geqslant k_{o}^{2}$. This implies (b) of the main Theorem. It remains to prove (c) for the family $\left(\mathcal{F}_{\alpha}^{4}\right)_{\alpha \in \overline{\mathbb{C}}}$. We need a definition.

Definition 2. - Let $G=\langle j . z, j . z+A\rangle$. We say that $b \in \mathbb{C} / \Gamma$ is generic for $G$, if for any $f \in G \backslash\{i d\}$ the point $b$ is not a fixed point of $f$. We observe that the set of points $b \in \mathbb{C} / \Gamma$ which are not generic for $G$ is countable. In particular, the set of generic points is dense and non countable in a fundamental domain of $\Gamma$.

Let $\alpha \in \mathbb{C}$ and $L$ be a leaf of $\mathcal{F}_{\alpha}^{4}$. We say that $L$ is generic, if

(i) $L \not \subset \mathcal{L}$

(ii) Let $\tilde{L}$ be a leaf of $\tilde{\mathcal{F}}_{\alpha}$ such that $\Pi(\tilde{L})=L$. Then $T_{a} \cap \tilde{L}$ contains a point $b$ which is generic for $G_{\alpha}$.

We will say also that $\tilde{L}$ is generic. We observe that condition (ii) does not depend on the point $b \in T_{a} \cap \tilde{L}$. Moreover, for all $\alpha \in \mathbb{C}$, the set of generic leaves of $\tilde{\mathcal{F}}_{\alpha}$ contains a non countable and dense set of leaves. We would like to observe also that, the leaves of $\tilde{\mathcal{F}}_{\alpha}$ which contain the local separatrices of the saddle points of this foliation are not generic (see the proof of (b) of Proposition 4). Assertion (c) of the main Theorem will follow from the next result.

PROPOSITION 6. - Let $\tilde{L}$ be a generic leaf of $\tilde{\mathcal{F}}_{\alpha}$. Then there exists a holomorphic covering map $\pi: \tilde{L} \rightarrow \mathbb{C} / \Gamma$. In particular, if $\alpha \in E$ then the generic leaf of $\mathcal{F}_{\alpha}^{4}$ has genus one.

Proof. - We will use (a) of Proposition 4 and the first integral $H_{1}$ of $\mathcal{F}_{1}^{4}$ in the proof of this proposition. Let $\tilde{L}_{1}$ be a generic leaf of $\tilde{\mathcal{F}}_{1}$. According to (a) of Proposition $4, \tilde{L}_{1}$ is biholomorphic to $\mathbb{C} / \Gamma$. Let $V=M \backslash h^{-1}\{0,1, \infty\}$ be as before. Denote by $L_{1}$ and $L$ the intersections of $\tilde{L}_{1}$ and $\tilde{L}$ with $V$. We know that $h_{1}:=\left.h\right|_{L_{1}}: L_{1} \rightarrow W$ and $h_{o}:=\left.h\right|_{L}: L \rightarrow W$ are covering maps, where $W=\overline{\mathbb{C}} \backslash\{0,1, \infty\}$. Moreover, the fiber $h_{1}^{-1}(z)$ of a point $z \in W$ has three points in $L_{1}$ (see (c.1) in the proof of Proposition 4). Let us construct $\left.\pi\right|_{L}: L \rightarrow L_{1}$ in such a way that $h_{o}=h_{1} \circ \pi$.

Fix a point $b_{1} \in h_{1}^{-1}(a) \subset T_{a} \cap L_{1}$ and a point $b_{o} \in h_{o}^{-1}(a) \subset T_{a} \cap L$ and set $\pi\left(b_{o}\right)=b_{1}$. Given $m \in L$, fix a path $\tilde{\gamma}_{m}:[0,1] \rightarrow L$ such that $\tilde{\gamma}_{m}(0)=b_{o}$ and $\tilde{\gamma}_{m}(1)=m$. Let 
$\gamma_{m}=h_{o} \circ \tilde{\gamma}_{m}:[0,1] \rightarrow W$ and $\hat{\gamma}_{m}:[0,1] \rightarrow L_{1}$ be the lifting of $\gamma_{m}$ by $h_{1}$ in $L_{1}$, such that $\hat{\gamma}_{m}(0)=b_{1}$. In order to define $\pi(m)$ it is enough to prove the following:

ASSERTION 1. - The final point of $\hat{\gamma}_{m}, \hat{\gamma}_{m}(1)$, does not depend on the path $\tilde{\gamma}_{m}$. Therefore, we can define $\pi(m)=\hat{\gamma}_{m}(1)$.

Proof. - It is enough to prove the assertion in the case where the path $\tilde{\gamma}_{m}$ is closed, that is $\tilde{\gamma}_{m}(0)=\tilde{\gamma}_{m}(1)=b_{o}$. In this case, since $\tilde{L}$ is generic for $\tilde{\mathcal{F}}_{\alpha}$, the global holonomy of the path $\gamma_{m}=h_{o} \circ \tilde{\gamma}_{m}$ in $G_{\alpha}$ is the identity. In fact, if we denote this holonomy by $k \in G_{\alpha}$, then $k\left(b_{o}\right)=\tilde{\gamma}_{m}(1)=b_{o}$. Therefore, the definition implies that $k=i d$. Now, write $\gamma_{m}$ as a word in $\gamma_{1}$ and $\gamma_{2}, \gamma_{m}=\gamma_{1}^{m_{1}} \circ \gamma_{2}^{n_{1}} \circ \cdots \circ \gamma_{1}^{m_{r}} \circ \gamma_{2}^{n_{r}}$. We have

$$
k(z)=f_{\alpha}^{m_{1}} \circ g_{\alpha}^{n_{1}} \circ \cdots \circ f_{\alpha}^{m_{r}} \circ g_{\alpha}^{n_{r}}(z)=c . z+e,
$$

where $c=j^{m_{1}+n_{1}+\cdots+m_{r}+n_{r}}$. Since $c=1$, it follows that 3 divides $m_{1}+n_{1}+\cdots+m_{r}+n_{r}$. Now, let $K$ be the global holonomy of $\gamma_{m}$ with respect to the foliation $\tilde{\mathcal{F}}_{1}$. As we have seen in (c.1) of the proof of Proposition 4, $G_{1}=\langle j . z, j . z\rangle$, so that $K(z)=j^{m_{1}+n_{1}+\cdots+m_{r}+n_{r}} . z=z$. This implies that $\hat{\gamma}_{m}$ is closed and the assertion.

Now, we have to extend $\pi$ to $\tilde{L} \cap h^{-1}\{0,1, \infty\}$. To do this, we first observe that $\tilde{L}_{1} \cap h^{-1}(c)$ contains exactly one point, for all $c \in\{0,1, \infty\}$, say $p(c)$. Moreover, $\tilde{L}_{1}$ and $\tilde{L}$ intersect transversely $h^{-1}(c)$ (Proposition 3).

In fact, let us consider, for instance, the case $c=1$. Since $h^{-1}(1)=D_{1} \cup \tilde{\ell}_{1} \cup \tilde{\ell}_{2} \cup \tilde{\ell}_{3}$ and $\tilde{\ell}_{1} \cup \tilde{\ell}_{2} \cup \tilde{\ell}_{3}$ is invariant for $\tilde{\mathcal{F}}_{1}$ and $\tilde{\mathcal{F}}_{\alpha}$, we must have, $\tilde{L}_{1} \cap h^{-1}(1) \subset D_{1}$ and $\tilde{L} \cap h^{-1}(1) \subset D_{1}$. On the other hand, $\tilde{L}_{1}=\tilde{h}_{1}^{-1}(e)$, where $\tilde{h}_{1}=H_{1} \circ \Pi$ and $e \in \mathbb{C}$. In the chart $(u, x) \in M$, such that $\Pi(u, x)=(x, u . x)$, we have

$$
\tilde{h}_{1}(u, x)=H_{1} \circ \Pi(u, x)=\frac{\left(x-j^{2}\right)(u x-j)\left(u-j^{2}\right)}{(x-j)\left(u x-j^{2}\right)(u-j)} \Rightarrow \tilde{h}_{1}(u, 0)=\frac{u-j^{2}}{u-j}
$$

and so $\tilde{L}_{1} \cap D_{1}=\left(\frac{j e-j^{2}}{1-e}, 0\right)=p(0)$. Hence, we can define $\pi$ in $\tilde{L} \cap h^{-1}(1)$, by $\pi(q)=p(0)$ for all $q \in \tilde{L} \cap h^{-1}(1)$. In the same way, we can extend $\pi$ to $\tilde{L} \cap h^{-1}(0)$ and $\tilde{L} \cap h^{-1}(\infty)$, obtaining a map

$$
\pi: \tilde{L} \rightarrow \tilde{L}_{1}
$$

which is holomorphic in $L=\tilde{L} \backslash h^{-1}\{0,1, \infty\}$.

ASSERTION 2. $-\pi$ is holomorphic in $\tilde{L}$. Moreover, it is a local homeomorphism in a neighborhood of each point $q \in \tilde{L} \backslash L$.

Proof. - Let $q \in \tilde{L} \cap h^{-1}\{0,1, \infty\}$. We can suppose without lost of generality that $q \in$ $\tilde{L} \cap h^{-1}(1)$, so that $q \in \tilde{L} \cap D_{1}$. Let us consider the case where $q$ is in the domain of the chart $(u, x)$ in which the blowing up at $0 \in \mathbb{C}^{2}$ is written as $\Pi(u, x)=(x, u x)$. In this chart, we have $q=\left(u_{o}, 0\right), p(0)=\left(a_{o}, 0\right)=\left(\frac{j e-j^{2}}{1-e}, 0\right)$ and

$$
h(u, x)-1=\frac{x^{3}\left(1-u^{3}\right)}{1-x^{3}} .
$$

Since $\tilde{L}$ and $\tilde{L}_{1}$ intersect transversely $D_{1}$ we can parametrize these leaves in a neighborhood of $q$ and $p(0)$ as $x \mapsto(u(x), x)$ and $x \mapsto\left(u_{1}(x), x\right)$ respectively, where $u, u_{1}: D(0, r) \rightarrow \mathbb{C}$ are holomorphic and $u_{o}=u(0), a_{o}=u_{1}(0)$. It follows from (16) that there are local charts $z$ and $w$ 
in $\tilde{L}$ and $\tilde{L}_{1}$, around $q$ and $p(0)$, respectively, such that $z(q)=w(p(0))=0$ and $h_{o}(z)=1+z^{3}$ and $h_{1}(w)=1+w^{3}$. Since $h_{o}=h_{1} \circ \pi$ outside $D_{1} \cap \tilde{L}$, we get

$$
z^{3}=(w(\pi(z)))^{3} \forall z \neq 0 \Rightarrow w(\pi(z))=\delta . z \text { where } \delta^{3}=1 .
$$

Clearly (17) implies the assertion.

Now, in order to prove that $\pi: \tilde{L} \rightarrow \tilde{L}_{1}$ is a covering map, we use the so-called "property of the unique lifting of paths" (cf. [16]):

"Let $f: X \rightarrow Y$ be a local homeomorphism, where $X$ and $Y$ are connected manifolds. Then, $f$ is a covering map if, and only if, it has the property of the unique lifting of paths, that is, given a path $\gamma:[0,1] \rightarrow Y$ such that $\gamma(0)=y_{o} \in Y$ and $x_{o} \in X$ such that $f\left(x_{o}\right)=y_{o}$, then there exists a unique path $\tilde{\gamma}:[0,1] \rightarrow X$ such that $\tilde{\gamma}(0)=x_{o}$ and $f \circ \tilde{\gamma}=\gamma$."

It is not very difficult to verify that $\pi: \tilde{L} \rightarrow \tilde{L}_{1}$ satisfies the property of the unique lifting of paths. We leave the proof of this fact for the reader. This proves Proposition 6.

Let us prove assertion (c) of the main Theorem for the family $\left(\mathcal{F}_{\alpha}^{4}\right)_{\alpha \in \overline{\mathbb{C}}}$. Let $\alpha \notin E$ and $\tilde{L}$ be a generic leaf of $\tilde{\mathcal{F}}_{\alpha}$. Let $\pi: \tilde{L} \rightarrow \tilde{L}_{1} \simeq \mathbb{C} / \Gamma$ be the covering of Proposition 6 . Since $\tilde{L}$ is not compact (see (c) of the Corollary of Proposition 5), we must have $\tilde{L} \simeq \mathbb{C}$ or $\tilde{L} \simeq \mathbb{C}^{*}$. Since the holomorphic universal covering of $\mathbb{C}^{*}$ is $\mathbb{C}$, in both cases there exists a holomorphic non-constant map $\tilde{f}: \mathbb{C} \rightarrow \tilde{L}$. Hence $f=\Pi \circ \tilde{f}: \mathbb{C} \rightarrow L=\Pi(\tilde{L})$ is a non-constant holomorphic map such that $f(\mathbb{C}) \subset L \cup \operatorname{sing}\left(\mathcal{F}_{\alpha}^{4}\right)$, which proves (c) of the main Theorem.

It remains to prove that a generic leaf of $\tilde{\mathcal{F}}_{\alpha}$ is biholomorphic to $\mathbb{C}$, as asserted in Remark 3 . Let us consider the automorphisms of the covering $\pi ; A u t(\pi)=\{k \in A u t(\tilde{L}) ; \pi \circ k=\pi\}$. We have two possibilities: either (i) $\tilde{L} \simeq \mathbb{C}^{*}$ and $A u t(\pi) \simeq \mid \mathbb{Z}$, or (ii) $\tilde{L} \simeq \mathbb{C}$ and $A u t(\pi) \simeq \mathbb{Z}^{2}$. We will prove that $A u t(\pi) \simeq \mathbb{Z}^{2}$.

First of all, fix a point $b_{1} \in \tilde{L}_{1}$ and a point $b \in \tilde{L}$, such that $h\left(b_{1}\right)=h(b)=a \in W=$ $\overline{\mathbb{C}} \backslash\{0,1, \infty\}$. Recall that for any class of closed curve $[\delta] \in \Pi_{1}\left(\tilde{L}_{1}, b_{1}\right)$ there exists $k_{\delta} \in \operatorname{Aut}(\pi)$ such that, if $\tilde{\delta}$ is the lifting of d by $\pi$ such that $\tilde{\delta}(0)=b$, then $\tilde{\delta}(1)=k_{\delta}(b)$. Now, consider the curves $\gamma_{2} \circ \gamma_{1}^{2}$ and $\gamma_{1} \circ \gamma_{2} \circ \gamma_{1}$ in $W$. The lifting of each one of these curves by $h_{1}$ in $\tilde{L}_{1}$ is a closed curve, because $g_{1} \circ f_{1}^{2}=f_{1} \circ g_{1} \circ f_{1}=i d_{T_{a}}$. Let us denote by $\delta_{1}$ and $\delta_{2}$, respectively, these liftings (where $\delta_{1}(0)=\delta_{2}(0)=b_{1}$ ), by $\tilde{\delta}_{1}$ and $\tilde{\delta}_{2}$ the liftings of $\delta_{1}$ and $\delta_{2}$ by $\pi$ (where $\left.\tilde{\delta}_{1}(0)=\tilde{\delta}_{2}(0)=b\right)$ and by $k_{1}$ and $k_{2}$ the associate elements of Aut $(\pi)$. Since $h_{o}=h_{1} \circ \pi, \tilde{\delta}_{1}$ and $\tilde{\delta}_{2}$ coincide with the liftings of $\gamma_{2} \circ \gamma_{1}^{2}$ and $\gamma_{1} \circ \gamma_{2} \circ \gamma_{1}$, respectively, by $h_{1}$. It follows that

$$
\begin{aligned}
& k_{1}(b)=\tilde{\delta}_{1}(1)=g_{\alpha} \circ f_{\alpha}^{2}(b)=b+A(\bmod (\Gamma)) \quad \text { and } \\
& k_{2}(b)=\tilde{\delta}_{2}(1)=f_{\alpha} \circ g_{\alpha} \circ f_{\alpha}(b)=b+j \cdot A(\bmod (\Gamma)),
\end{aligned}
$$

where $A=A(\alpha)$. Now, suppose by contradiction that $A u t(\pi) \simeq \mathbb{Z}$. If $k$ is a generator of $A u t(\pi)$, then $k_{1}=k^{m}$ and $k_{2}=k^{n}$ for $m, n \in \mathbb{Z}$, so that $k_{1}^{n}=k_{2}^{m}$. But, this implies that

$$
b+n \cdot A(\bmod (\Gamma))=\left(g_{\alpha} \circ f_{\alpha}^{2}\right)^{n}(b)=\left(f_{\alpha} \circ g_{\alpha} \circ f_{\alpha}\right)^{m}(b)=b+m j \cdot A(\bmod (\Gamma))
$$

so that

$$
(n-m j) . A \in \Gamma \Rightarrow\left(n^{2}+m^{2}+m n\right) A \in \Gamma
$$

as the reader can check. This contradiction finishes the proof of Remark 3.

\subsection{The family of degree three}

In order to construct the family of degree three, we observe that the equations in (8) and (9), which define the family $\left(\mathcal{F}_{\alpha}^{4}\right)_{\alpha \in \overline{\mathbb{C}}}$, are symmetric with respect to the change of variables 
$S(x, y)=(y, x)$. It is possible to prove that any foliaton with this property is the pull-back of another foliation by the map $T(x, y)=(x+y, x . y)$. In our case, the vector field which defines the foliation $\mathcal{F}_{\alpha}^{4}$ is $\widehat{Z}_{\alpha}=\widehat{X}-\alpha \widehat{Y}$, where

$$
\widehat{X}=x\left(x^{3}-1\right) \frac{\partial}{\partial x}+y\left(y^{3}-1\right) \frac{\partial}{\partial y} \quad \text { and } \quad \widehat{Y}=y^{2}\left(x^{3}-1\right) \frac{\partial}{\partial x}+x^{2}\left(y^{3}-1\right) \frac{\partial}{\partial y} .
$$

As the reader can check, we have $\widehat{X}=T^{*}(X)$ and $\widehat{Y}=T^{*}(Y)$, where

$$
X(u, v)=\left(-u+2 v^{2}-4 u^{2} v+u^{4}\right) \frac{\partial}{\partial u}+v\left(-2-3 u v+u^{3}\right) \frac{\partial}{\partial v},
$$

and

$$
Y(u, v)=\left(2 v-u^{2}+u v^{2}\right) \frac{\partial}{\partial u}+\left(3 u v-u^{3}+2 v^{3}\right) \frac{\partial}{\partial v},
$$

so that $\widehat{Z}_{\alpha}=T^{*}\left(Z_{\alpha}\right)$, where $Z_{\alpha}=X-\alpha Y$.

Observe that, although the vector field $Z_{\alpha}$ has degree four, its homogeneous part of degree four is $u^{4} \frac{\partial}{\partial u}+v u^{3} \frac{\partial}{\partial v}=u^{3}\left(u \frac{\partial}{\partial u}+v \frac{\partial}{\partial v}\right)$, so that the foliation defined by $Z_{\alpha}$ has degree three. We will denote this foliation by $\mathcal{F}_{\alpha}^{3}$. The foliation $\mathcal{F}_{\infty}^{3}$ will be the foliation defined by the vector field $Y$, which is also of degree three.

In order to study the foliation $\mathcal{F}_{\alpha}^{3}$, let us see first how the rational map $T$ transforms the lines of the configuration $\mathcal{C}$. Observe that:

(I) The lines $\left(x=j^{k}\right)$ and $\left(y=j^{k}\right)$ are sent by $T$ into the line $\left(v=j^{k} u-j^{2 k}\right), k=0,1,2$. This implies that the foliation $\mathcal{F}_{\alpha}^{3}$ has three invariant lines; $\ell_{k}:=\left(v=j^{k} u-j^{2 k}\right)$, $k=0,1,2$.

(II) The line $(y=x)$ is sent by $T$ into the conic $C_{1}:=\left(v=\frac{1}{4} u^{2}\right)$.

(III) The lines $(y=j x)$ and $\left(y=j^{2} x\right)$ are sent by $T$ into the conic $C_{2}:=\left(v=u^{2}\right)$.

It follows from (I), (II) and (III) that, for all $\alpha \in \overline{\mathbb{C}}, \mathcal{F}_{\alpha}^{3}$ leaves invariant an algebraic curve of degree 7 , which is the union of five rational curves: three lines, $\ell_{k}, k=0,1,2$, and two conics $C_{1}$ and $C_{2}$. The conics are tangent in the points $q_{1}=[0: 0: 1]$ and $q_{2}=[0: 1: 0]$. The lines $\ell_{k}$, $k=0,1,2$, intersect in the points $p_{01}=\left[-j^{2}: j: 1\right] \in \ell_{0} \cap \ell_{1}, p_{02}=\left[-j: j^{2}: 1\right] \in \ell_{0} \cap \ell_{2}$ and $p_{12}=[-1: 1: 1] \in \ell_{1} \cap \ell_{2}$. Observe that $p_{01}, p_{02}, p_{12} \in C_{2}$. Finally, the lines are tangent to the conic $C_{1}$ in the points $p_{0}=[2: 1: 1] \in \ell_{0} \cap C_{1}, p_{1}=\left[2 j: j^{2}: 1\right] \in \ell_{1} \cap C_{1}$ and $p_{2}=\left[2 j^{2}: j: 1\right] \in \ell_{2} \cap C_{1}$. In Fig. 4 , we sketch the five curves and the points.

Since the five curves are invariant for $\mathcal{F}_{\alpha}^{3}$, for all $\alpha \in \overline{\mathbb{C}}$, it follows that all points in the intersections of the curves are singularities for $\mathcal{F}_{\alpha}^{3}$. As we will see, if $\alpha \notin\left\{0,1, j, j^{2}, \infty\right\}$, then the foliation $\mathcal{F}_{\alpha}^{3}$ has five more singularities, one in each curve of the configuration sketched in Fig. 4. In the next result we precise how the family of foliations $\left(\mathcal{F}_{\alpha}^{3}\right)_{\alpha \in \overline{\mathbb{C}}}$ looks like.

PROPOSITION 7.-Assertions (a), (b) and (c) of the main Theorem are true for the family $\left(\mathcal{F}_{\alpha}^{3}\right)_{\alpha \in \overline{\mathbb{C}}}$. Moreover, the set $A^{3}$ of (a) is $\left\{0,1, j, j^{2}, \infty\right\}$ and if $\alpha \notin A^{3}$ then the singularities of $\mathcal{F}_{\alpha}^{3}$ are of the following local analytic types:

(a.1) The eight points in the intersection of the five curves are dicritical; $p_{01}, p_{02}$ and $p_{12}$ are of radial type and in a neighborhood of each of the points $q_{1}, q_{2}, p_{0}, p_{1}$ and $p_{2}, \mathcal{F}_{\alpha}^{3}$ has a meromorphic first integral of the type $z^{2} / w=$ cte.

(a.2) Each of the five curves contains another singularity, say $P_{1}(\alpha) \in C_{1}, P_{2}(\alpha) \in C_{2}$ and $Q_{k}(\alpha) \in \ell_{k}, k=0,1,2$. Moreover, $\mathcal{F}_{\alpha}^{3}$ has a local holomorphic first integral in a neighborhood of each of these singularities, of the following types: $z^{6} . w=$ cte in a neighborhood of $P_{1}(\alpha) \in C_{1}$ and $z^{3} . w=$ cte in a neighborhood of the others. 


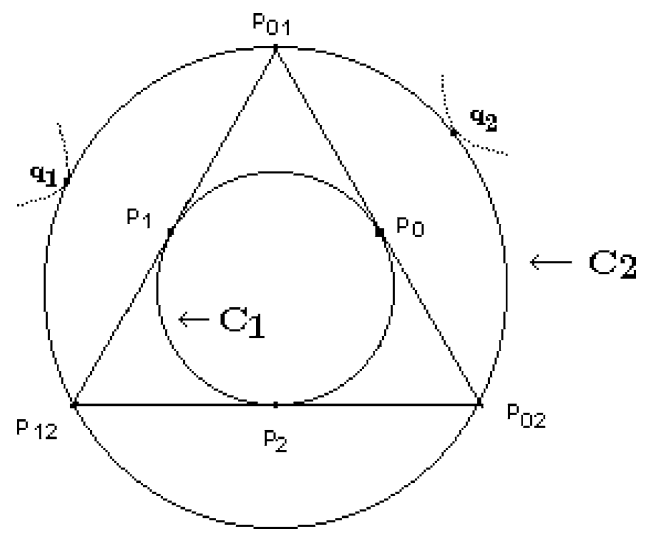

Fig. 4.

Proof. - We observe that most of the above assertions follow from the analysis that we have done for the family $\mathcal{F}_{\alpha}^{4}$. So, for instance, the fact that if $\alpha \in E$ then $\mathcal{F}_{\alpha}^{4}$ has a first integral, implies that all leaves of $\mathcal{F}_{\alpha}^{3}$ are algebraic, and so it has a rational first integral, by Darboux's Theorem.

Let us prove that $A^{3}=\left\{0,1, j, j^{2}, \infty\right\}$ and assertions (a.1) and (a.2). Observe first that the eight intersection points of the five curves are singularities of $\mathcal{F}_{\alpha}^{3}$. Moreover, in each of the five invariant curves, $\mathcal{F}_{\alpha}^{3}$ has a unique singularity contained in $T\left\{q_{1}(\alpha), \ldots, q_{9}(\alpha)\right\}$ (see Proposition 2). These singularities are:

$$
\begin{aligned}
& T\left(\frac{1}{\alpha}, \frac{1}{\alpha}\right)=\left(\frac{2}{\alpha}, \frac{1}{\alpha^{2}}\right):=P_{1}(\alpha) \in C_{1}, \\
& T\left(\frac{j}{\alpha}, \frac{j^{2}}{\alpha}\right)=T\left(\frac{j^{2}}{\alpha}, \frac{j}{\alpha}\right)=\left(\frac{-1}{\alpha}, \frac{1}{\alpha^{2}}\right):=P_{2}(\alpha) \in C_{2}, \\
& T(1, \alpha)=T(\alpha, 1)=(1+\alpha, \alpha):=Q_{0}(\alpha) \in \ell_{0}, \\
& T\left(j, j^{2} \alpha\right)=T\left(j^{2} \alpha, j\right)=\left(j+j^{2} \alpha, \alpha\right):=Q_{1}(\alpha) \in \ell_{1}
\end{aligned}
$$

and

$$
T\left(j^{2}, j \alpha\right)=T\left(j \alpha, j^{2}\right)=\left(j^{2}+j \alpha, \alpha\right):=Q_{2}(\alpha) \in \ell_{2} .
$$

If $\alpha \notin\left\{0,1, j, j^{2}, \infty\right\}$, then these singularities do not coincide with the points in the intersections of the curves. Therefore, in this case, $\mathcal{F}_{\alpha}^{3}$ has $8+5=13=3^{2}+3+1$ distinct singularities, which implies that all singularities are non-degenerate.

In order to prove (a.1) and (a.2), we will study the map $T$ near the points of $\operatorname{sing}\left(\mathcal{F}_{\alpha}^{4}\right)$. In homogeneous coordinates, we can write $T(X, Y, Z)=\left(Z(X+Y), X . Y, Z^{2}\right)=(U, V, W)$. It follows that $T$ is not defined in the points $[1: 0: 0]$ and $[0: 1: 0]$. Moreover, the singular set of $T$ is $S=(Y=X) \cup(Z=0)$, where $(Y=X)$ is the ramification set of $T$ and $T(Z=0)=$ $[0: 1: 0] \in C_{1} \cap C_{2}$. Remark also that:

(i) $T(Y=X)=C_{1}$.

(ii) If $m \in \mathbb{C P}(2) \backslash C_{1}$, then $T^{-1}(m)$ contains two points, say $m_{1}$ and $m_{2}$. Moreover $T$ is a local biholomorphism in a neighborhood of $m_{k}, k=1,2$. 
(iii) If $m \in C_{1} \backslash\{[0: 1: 0]\}=\left\{(u, v) \in \mathbb{C}^{2} ; v=\frac{1}{4} u^{2}\right\}$, say $m_{o}=\left(u_{o}, \frac{1}{4} u_{o}^{2}\right)$, then $T^{-1}\left(m_{o}\right)=\left\{\left(\frac{1}{2} u_{o}, \frac{1}{2} u_{o}\right)\right\}=\{m\}$. Moreover, there exists a holomorphic coordinate system, $\left(W_{1},\left(z_{1}, w_{1}\right)\right)$ in a neighborhood of $m$ and $\left(W_{2},\left(z_{2}, w_{2}\right)\right)$ in a neighborhood of $m_{o}$, such that $z_{1}(m)=w_{1}(m)=0, z_{2}\left(m_{o}\right)=w_{2}\left(m_{o}\right)=0, W_{1} \cap(Y=X)=\left(w_{1}=0\right)$, $W_{2} \cap C_{1}=\left(w_{2}=0\right)$ and $T\left(z_{1}, w_{1}\right)=\left(z_{1}, w_{1}^{2}\right)$.

It follows from (ii) that for any singularity $p$ of $\mathcal{F}_{\alpha}^{3}$, which is not in $C_{1}$, there is a singularity $q$ of $\mathcal{F}_{\alpha}^{4}$ such that $T(q)=p$. Moreover, $\mathcal{F}_{\alpha}^{3}$ and $\mathcal{F}_{\alpha}^{4}$ are locally equivalent at $p$ and $q$. Using this fact, it is not difficult to see that:

(iv) $p_{01}, p_{02}$ and $p_{12}$ are radial singularities for $\mathcal{F}_{\alpha}^{3}$.

(v) $\mathcal{F}_{\alpha}^{3}$ has a local holomorphic first integral of the type $z^{3} . w=$ cte, in a neighborhood of each one of the singularities $P_{2}(\alpha), Q_{0}(\alpha), Q_{1}(\alpha)$ and $Q_{2}(\alpha)$.

The next result will allow us to study the dicritical singularities of $\mathcal{F}_{\alpha}^{3}$ which are contained in $C_{1}$.

LEMMA 1. - Let $Z(x, y)=p(x, y) \frac{\partial}{\partial x}+q(x, y) \frac{\partial}{\partial y}$ be a holomorphic vector field defined in a neighborhood of $0 \in \mathbb{C}^{2}$. Suppose that:

(a) 0 is a non-degenerate singularity of $Z$ and the characteristic numbers of $Z$ at 0 are rational and positive, say $p / q$ and $q / p$, where $p, q \in \mathbb{N}$ are relatively primes.

(b) Either $p, q \geqslant 2$ or $Z$ has at least two distinct local analytic separatrices through 0 .

Then there exists a holomorphic coordinate system $(W,(u, v))$ with $0 \in W, u(0)=v(0)=0$, in which $Z$ can be written as

$$
Z(u, v)=k\left(q \cdot u \frac{\partial}{\partial u}+p \cdot v \frac{\partial}{\partial v}\right)
$$

where $k \in \mathbb{C}^{*}$. In particular, $\frac{u^{p}}{v^{q}}$ is a meromorphic first integral of $Z$ in a neighborhood of 0 .

Proof. - If $p, q \geqslant 2$ then $Z$ has no resonance at 0 and the Poincaré's linearization Theorem implies that $Z$ is linearizable (cf. [2]). Therefore, we can suppose that $p=1$ and $q \geqslant 1$. In this case, Dulac's normal form (cf. [2]) implies that in a suitable coordinate system $(W,(u, v))$ such that $0 \in W$ and $u(0)=v(0)=0$, the vector field $Z$ can be written as

$$
Z(u, v)=k\left(u \frac{\partial}{\partial u}+\left(q v+b \cdot u^{q}\right) \frac{\partial}{\partial v}\right)
$$

where $k \in \mathbb{C}^{*}$. Let us prove that hypothesis (b) implies that $b=0$. Observe that, if this is the case, then $v / u^{q}$ is a local meromorphic first integral of $Z$.

Suppose by contradiction that $b \neq 0$. In this case, the function $F: \mathbb{C}^{2} \backslash(u=0) \rightarrow \mathbb{C}$ given by

$$
F(u, v)=u \cdot \exp \left(-\frac{v}{b \cdot u^{q}}\right),
$$

is a first integral of $Z$, as the reader can check. Observe that $Z$ has a holomorphic local analytic separatrix through $0:(u=0)$. If it had another local separatrix through 0 , then it would be contained in a level curve of $F$, say $(F=c)$, where $c \neq 0$. But $(u=0)$ is an essential singularity of $F$, and so these level curves are not analytic at $0 \in \mathbb{C}^{2}$.

Corollary. - Let $Z(x, y)=p(x, y) \frac{\partial}{\partial x}+q(x, y) \frac{\partial}{\partial y}$ be a holomorphic vector field defined in a neighborhood of $0 \in \mathbb{C}^{2}$. Suppose that 0 is a non-degenerate singularity of $Z$ and that $Z$ has two smooth invariant separatrices through 0 , with a tangency of order two at 0 . Then the 
characteristic numbers of $Z$ at 0 are 2 and $1 / 2$ and $Z$ has a local meromorphic first integral of the form $z^{2} \cdot w=$ cte.

Proof. - It is sufficient to prove that the characteristic numbers of $Z$ at 0 are 2 and $1 / 2$. Let $\gamma_{1}, \gamma_{2}$ be the smooth invariant separatrices through 0 . Since they have a tangency of order two at 0 , there is a holomorphic coordinate system $(W,(u, v))$ such that $0 \in W, u(0)=v(0)=0$, $\gamma_{1} \cap W=(v=0)$ and $\gamma_{2} \cap W=\left(v-u^{2}=0\right)$. Since $(v=0)$ is invariant for $Z$, in this coordinate system $Z$ can be written as

$$
Z(u, v)=\left(a u+b v+r_{1}(u, v)\right) \frac{\partial}{\partial u}+v\left(c+r_{2}(u, v)\right) \frac{\partial}{\partial v}
$$

where the linear part of $Z$ at 0 is $(a u+b v) \frac{\partial}{\partial u}+c \cdot v \frac{\partial}{\partial v}$ and the characteristic numbers are $c / a$, $a / c$. Now, since $\gamma_{2}$ is invariant for $Z$, we must have

$$
Z\left(v-u^{2}\right)=-2 u\left(a u+b v+r_{1}(u, v)\right)+v\left(c+r_{2}(u, v)\right)=f(u, v) \cdot\left(v-u^{2}\right),
$$

where $f$ is holomorphic. By comparing the two jets of $-2 u\left(a u+b v+r_{1}(u, v)\right)$ and $f(u, v)$. $\left(v-u^{2}\right)$ at 0 , it is not difficult to see that $c=f(0,0)$ and that $f(0,0)=2 a$. Therefore $c / a=2$.

The above result implies that $\mathcal{F}_{\alpha}^{3}$ has a meromorphic first integral of the form $\frac{z^{2}}{w}$ in a neighborhood of each one of the singularities $p_{0}, p_{1}, p_{2}, q_{1}$ and $q_{2}$, because each of those singularities belongs to $C_{1}$ and to another one of the four other invariant curves, and $C_{1}$ has a tangency of order two with the curve at the point (see Fig. 4).

We have already proved (a.1) of Proposition 7. In order to finish the proof of (a.2), it remains to prove that $\mathcal{F}_{\alpha}^{3}$ has a holomorphic first integral of the form $z^{6} . w=$ cte in a neighborhood of $P_{1}(\alpha) \in C_{1}$. We will use (iii). Since $T^{-1}\left(P_{1}(\alpha)\right)=q_{1}(\alpha) \in(Y=X)$, there exists a holomorphic coordinate system, $\left(W_{1},\left(z_{1}, w_{1}\right)\right)$ in a neighborhood of $m=q_{1}(\alpha)$ and $\left(W_{2},\left(z_{2}, w_{2}\right)\right)$ in a neighborhood of $m_{o}=P_{1}(\alpha)$, such that $z_{1}(m)=w_{1}(m)=0, z_{2}\left(m_{o}\right)=w_{2}\left(m_{o}\right)=0$, $W_{1} \cap(Y=X)=\left(w_{1}=0\right), W_{2} \cap C_{1}=\left(w_{2}=0\right)$ and $T\left(z_{1}, w_{1}\right)=\left(z_{1}, w_{1}^{2}\right)$. We assert that the characteristic numbers of $\mathcal{F}_{\alpha}^{3}$ at $P_{1}(\alpha)$ are -6 and $-1 / 6$.

Observe first that $\mathcal{F}_{\alpha}^{3}$ can be represented in the coordinate system $\left(z_{2}, w_{2}\right)$ by a differential equation of the form

$$
\left(a z_{2}+b w_{2}+r\left(z_{2}, w_{2}\right)\right) d w_{2}+6 w_{2} d z_{2}=0
$$

where the characteristic numbers are $-6 / a$ and $-a / 6$, because $\left(w_{2}=0\right)$ is invariant for $\mathcal{F}_{\alpha}^{3}$. Since $T\left(z_{1}, w_{1}\right)=\left(z_{1}, w_{1}^{2}\right)$, we get from $(18)$ that $\mathcal{F}_{\alpha}^{4}$ is represented in a neighborhood of $q_{1}(\alpha)$ by

$$
\begin{aligned}
& \left(a z_{1}+b w_{1}^{2}+r\left(z_{1}, w_{1}^{2}\right)\right) 2 w_{1} \cdot d w_{1}+6 w_{1}^{2} d z_{1}=0 \\
& \Longrightarrow\left(a z_{1}+b w_{1}^{2}+r\left(z_{1}, w_{1}^{2}\right)\right) d w_{1}+3 w_{1} d z_{1}=0 .
\end{aligned}
$$

By using the local form of $\mathcal{F}_{\alpha}^{4}$ in a neighborhood of $\left(\frac{1}{\alpha}, \frac{1}{\alpha}\right)=q_{1}(\alpha)$ (see (b.2) of Proposition 2), it follows that $a=1$, which proves that the characteristic numbers of $\mathcal{F}_{\alpha}^{3}$ at $P_{1}(\alpha)$ are -6 and $-1 / 6$. Moreover, after a linear change of coordinates, we can suppose that the linear part in (18) is $z_{2} d w_{2}+6 w_{2} d z_{2}=0$. We want to prove that the foliation $\mathcal{F}_{\alpha}^{3}$ is linearizable in a neighborhood of $P_{1}(\alpha)$, which means that $\mathcal{F}_{\alpha}^{3}$ can be represented by $z d w+6 w d z=0$, after a holomorphic local change of coordinates. In order to prove this fact we use the following: 
(vi) $\mathcal{F}_{\alpha}^{4}$ has a holomorphic first integral in a neighborhood of $q_{1}(\alpha)$ (Proposition 2).

(vii) If there is a neighborhood $V$ of $P_{1}(\alpha)$ such that the leaves of the restricted foliation $\left.\mathcal{F}_{\alpha}^{3}\right|_{V}$ are closed in $V$, then $\mathcal{F}_{\alpha}^{3}$ has a holomorphic first integral in (a possibly smaller) neighborhood $U$ of $P_{1}(\alpha)$ (cf. [18]). In particular, $\mathcal{F}_{\alpha}^{3}$ is linearizable in $U$.

Since $\mathcal{F}_{\alpha}^{4}=T^{*}\left(\mathcal{F}_{\alpha}^{3}\right)$, it is clear that (vi) $\Rightarrow$ (vii). This finishes the proof of (a.2).

Let us prove that the family $\left(\mathcal{F}_{\alpha}^{3}\right)_{\alpha \in \overline{\mathbb{C}}}$ satisfies (b) of the main Theorem. First of all, observe that the rational map

$$
R(u, v)=\frac{\left(u^{3}-3 u v\right)^{2}-4 v^{3}}{v^{3}-u^{3}+3 u v+1}
$$

is a first integral of $\mathcal{F}_{\infty}^{3}$, because

$$
\widehat{R}(x, y)=R \circ T(x, y)=\frac{\left(y^{3}-x^{3}\right)^{2}}{\left(y^{3}-1\right)\left(x^{3}-1\right)}=\frac{H^{2}}{H+1},
$$

where $H=H(x, y)=\frac{y^{3}-x^{3}}{x^{3}-1}$ is a first integral of $\mathcal{F}_{\infty}^{4}$. Let $\alpha \in E$. As we have seen in the beginning of the proof, $\mathcal{F}_{\alpha}^{3}$ has a rational first integral, say $R_{\alpha}$, which we suppose primitive (that is, with irreducible generic level curve) and of degree $d_{\alpha}^{\prime}$. It follows from Bézout's Theorem, that the generic level $\left(R_{\alpha}=c_{1}\right)$ cuts the generic level $(R=c)$ in $6 d_{\alpha}^{\prime}$ points, counted with multiplicities. On the other hand, the foliations $\mathcal{F}_{\infty}^{3}$ and $\mathcal{F}_{\alpha}^{3}$ are transversal outside the curve $C=\bigcup_{k=0}^{3} \ell_{k} \cup C_{1} \cup C_{2}$. This implies that the intersection number of $(R=c)$ and $\left(R_{\alpha}=c_{1}\right)$ in each point of $(R=c) \cap\left(R_{\alpha}=c_{1}\right) \backslash C$ is one. Let $k_{\alpha}=\#\left((R=c) \cap\left(R_{\alpha}=c_{1}\right) \backslash C\right)$, so that $6 d_{\alpha}^{\prime}>k_{\alpha}$. Now, observe that, $T^{-1}\left((R=c) \cap\left(R_{\alpha}=c_{1}\right) \backslash C\right)$ is contained in the intersection of two leaves of $\mathcal{F}_{\infty}^{4}$ with at most two algebraic leaves of $\mathcal{F}_{\alpha}^{4}$, but outside the set of lines $\mathcal{L}$. It follows from (ii) above, that $2 k_{\alpha}=\#\left(T^{-1}\left((R=c) \cap\left(R_{\alpha}=c_{1}\right) \backslash C\right)\right) \geqslant 2 \#\left(G_{\alpha}\right) \geqslant 2 k(A(\alpha))^{2}$. Therefore, $6 d_{\alpha}^{\prime} \geqslant k_{\alpha} \geqslant k(A(\alpha))^{2}$, where $k(A)$ is like in (15). This proves that the family $\left(\mathcal{F}_{\alpha}^{3}\right)_{\alpha \in \overline{\mathbb{C}}}$ satisfies (b) of the main Theorem.

Now, let us see how the resolution of the elements of the family looks like. We have seen that $\mathcal{F}_{\alpha}^{3}$ has eight dicritical singularities, three radial and five with local meromorphic first integrals of the type $\frac{z^{2}}{w}=c t e$. The resolution of each of these five singularities is done with two blowing-ups, as sketched in Fig. 5.

In Fig. 5, $\pi_{1}$ and $\pi_{2}$ are the two blowing-ups, $D_{1}$ and $D_{2}$ the two divisors, and $m$ is a singularity of $\pi^{*}\left(\mathcal{F}_{\alpha}^{3}\right)$, in which this foliation has a local holomorphic first integral of the type $z^{2} \cdot w=$ cte, where $\pi=\pi_{1} \circ \pi_{2}$.

We observe that $D_{1}$ is invariant for $\pi^{*}\left(\mathcal{F}_{\alpha}^{3}\right)$, whereas $D_{2}$ is not. It follows that the resolution of $\mathcal{F}_{\alpha}^{3}$ involves a total of 13 blowing-ups, one at each radial singularity and two at each singularity as in Fig. 5. We call $M_{1}$ the manifold obtained from $\mathbb{C P}(2)$ after these blowing-ups, and $\Pi_{1}: M_{1} \rightarrow \mathbb{C P}(2)$ the blowing-up map. We denote by $\hat{\mathcal{F}}_{\alpha}$ the strict transform of $\mathcal{F}_{\alpha}^{3}$ by $\Pi_{1}$.

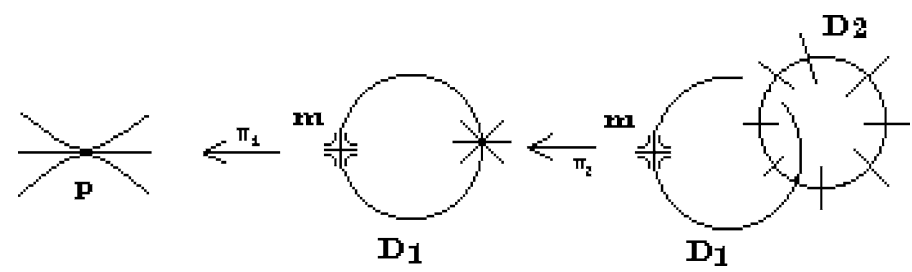

Fig. 5. 
Let $T_{1}: M-\rightarrow M_{1}$ be the map which makes the diagram below commute:

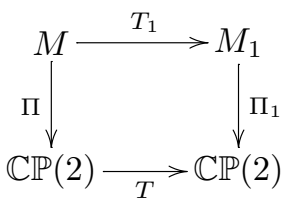

where $\Pi: M \rightarrow \mathbb{C P}(2)$ is the resolution of the foliation $\mathcal{F}_{\alpha}^{4}$.

Now, let us prove that the family satisfies (c) of the main Theorem and Remark 3 of $\S 1$. Let $\alpha \notin E$. If $L$ is a leaf of $\tilde{\mathcal{F}}_{\alpha}$ such that $L \not \subset \mathcal{L}$ and $L_{1}=T_{1}(L)$, then $L_{1}$ is a leaf of $\hat{\mathcal{F}}_{\alpha}$ and the restriction $\left.T_{1}\right|_{L}: L \rightarrow L_{1}$ extends to a holomorphic map $T_{L}: L \rightarrow L_{1}$, which is a ramified covering of degree at most two (see (ii) in the proof of Proposition 7). Moreover, since $L$ is a transcendent leaf of $\tilde{\mathcal{F}}_{\alpha}$, then $L_{1}$ is also transcendent. When $L$ is a generic leaf of $\tilde{\mathcal{F}}_{\alpha}$, then $L \simeq \mathbb{C}$ and we have a ramified covering $T_{L}: \mathbb{C} \simeq L \rightarrow L_{1}$ of degree at most two. This implies that $L_{1}$ is biholomorphic to $\mathbb{C}$ and finishes the proof of Proposition 7.

\subsection{The family of degree two}

The family of degree two is obtained from $\mathcal{F}_{\alpha}^{3}$ by a Cremona transformation, as illustrated in Fig. 6.

In this figure, we denote by $\pi_{1}: N \rightarrow \mathbb{C P}(2)$ the blowing-up at the three points $p_{01}, p_{02}$ and $p_{12}$. After this blowing-up process, we obtain three divisors, $D_{i j}=\pi_{1}^{-1}\left(p_{i j}\right)$, not invariant for the strict transform $\pi_{1}^{*}\left(\mathcal{F}_{\alpha}^{3}\right)$, because $p_{01}, p_{02}$ and $p_{12}$ are radial singularities $(\alpha \notin$ $\left.\left\{0,1, j, j^{2}, \infty\right\}\right)$. Moreover, the strict transforms of $\ell_{0}, \ell_{1}$ and $\ell_{2}$, say $\hat{\ell}_{0} \hat{\ell}_{1}$ and $\hat{\ell}_{2}$, have self-intersection -1 in $N$, so that we can blow down these three curves, obtaining another surface, say $N_{1}$, with three points, $J, K, L \in N_{1}$ and blowing-up map $\pi_{2}: N \rightarrow N_{1}$, such that $\pi_{2}^{-1}\{J, K, L\}=\hat{\ell}_{0} \cup \hat{\ell}_{1} \cup \hat{\ell}_{2}$. The map $\pi=\pi_{2} \circ\left(\pi_{1}\right)^{-1}$ is well defined, holomorphic and injective outside $\bigcup_{j} \ell_{j}$. This type of blowing-up-blowing-down process is known in the literature as a "Cremona transformation". It is well-known that the surface $N_{1}$, obtained after a Cremona transformation in $\mathbb{C P}(2)$, is again $\mathbb{C P}(2)$.

The curve indicated by $\widehat{C}_{1}$ in Fig. 6.2 is the strict transform of the curve $C_{1}$. This curve is sent by $\pi_{2}$ to the curve $Q$ of Fig. 6.3. This curve has degree four. In fact, the conics through the base points $p_{i j}$ of $\pi$ are sent by $\pi$ to straight lines. Since a generic conic passing through these points cuts $C_{1}$ in four points, its image by $\pi$ has intersection number four with $Q$, so that $Q$ has degree four. Observe that the curve $C_{2}$ is conic passing through the base points of $\pi$, therefore it is transformed by $\pi$ into a line, indicated by $R$ in Fig. 7. The quadric $Q$ has three cuspidal points,

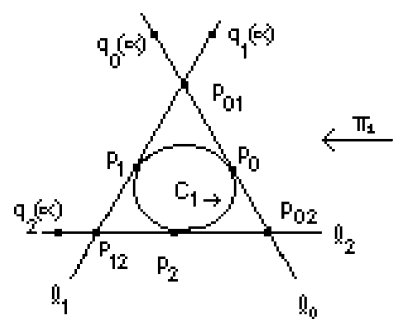

6.1

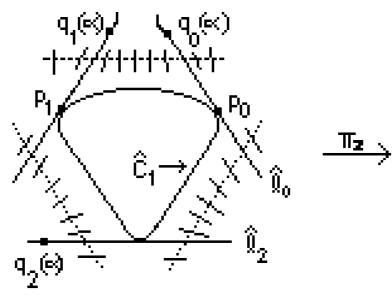

6.2

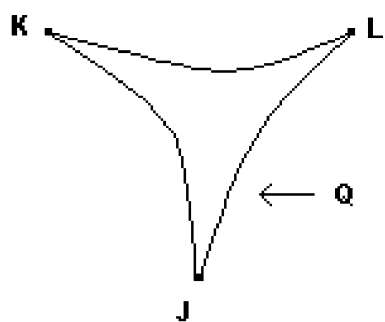

6.3

Fig. 6. 


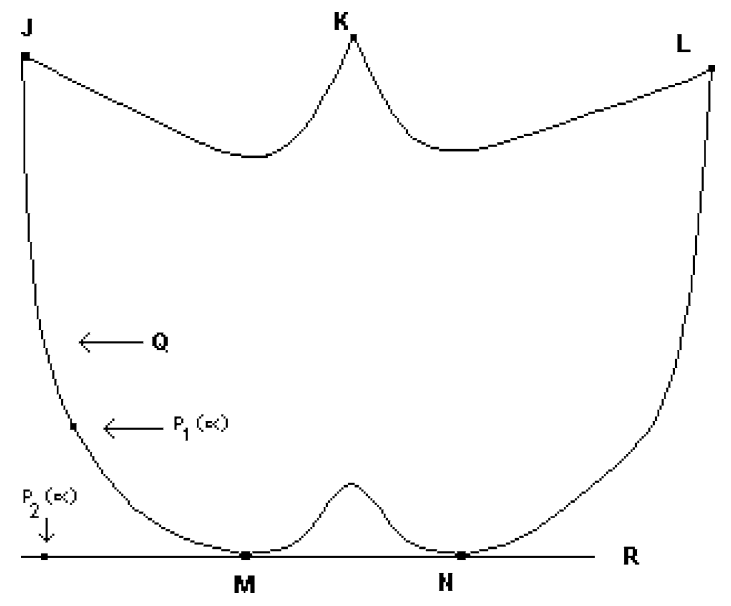

Fig. 7.

indicated by $J, K$ and $L$ in Fig. 6.3 and in Fig. 7. The line $R$ and the curve $Q$ are tangent in two points indicated by $M$ and $N$. Observe that $M=\pi\left(q_{1}\right)$ and $N=\pi\left(q_{2}\right)$ (or vice-versa), where $q_{1}$ and $q_{2}$ are the tangent points between $C_{1}$ and $C_{2}$.

Let $\mathcal{F}_{\alpha}^{2}$ be the strict transform of $\mathcal{F}_{\alpha}^{3}$ by $\pi^{-1}$. We will see that the foliation $\mathcal{F}_{\alpha}^{2}$ has degree two for all $\alpha \in \overline{\mathbb{C}}$, but first let us study the singularities of $\mathcal{F}_{\alpha}^{2}$ for $\alpha \notin\left\{0,1, j, j^{2}, \infty\right\}$.

We begin by observing that the foliation $\mathcal{F}_{\alpha}^{3}$ has four non-degenerate singularities outside $\bigcup_{k=0}^{2} \ell_{k}$ namely $q_{1}, q_{2} \in C_{1} \cap C_{2}, P_{1}(\alpha) \in C_{1}$ and $P_{2}(\alpha) \in C_{2}$ (see Fig. 4). These singularities are sent by $\pi$ into singularities of the same analytic type, because $\pi$ is a biholomorphism outside $\bigcup_{k=0}^{2} \ell_{k}$. After the three blowing-ups $\pi_{1}$, each of the invariant curves $\hat{\ell}_{k}$ contains two non-degenerate singularities, $p_{k}$ and $q_{k}(\alpha)$. This implies that after the blowing-down $\pi_{2}$, these curves are contracted in the three points $J, K$ and $L$, which are singularities of $\mathcal{F}_{\alpha}^{2}$. Therefore, the singularities of $\mathcal{F}_{\alpha}^{2}$ are $J, K, L$ and the four non-degenerate singularities $\pi\left\{q_{1}, q_{2}, P_{1}(\alpha), P_{2}(\alpha)\right\}$. We will use the same symbols to denote $P_{j}(\alpha)$ and $\pi\left(P_{j}(\alpha)\right), j=1,2$. Since $\pi\left(q_{1}\right)=M, \pi\left(q_{2}\right)=N$ and $\pi\left(P_{2}(\alpha)\right)=P_{2}(\alpha)$ are non-degenerate, belong to line $R$ and this line contains no other singularity of $\mathcal{F}_{\alpha}^{2}$, it follows that this foliation has degree two (see [17]). This implies that $J, K$ and $L$ are also non-degenerate because if a foliation of degree two has $7=2^{2}+2+1$ singularities, then these singularities are non-degenerate. Since $\pi$ is a biholomorphism outside $\bigcup_{k=0}^{2} \ell_{k}$, we have already proved that $\mathcal{F}_{\alpha}^{2}$ has the following local first integrals $\left(\alpha \notin\left\{0,1, j, j^{2}, \infty\right\}\right)$ :

(i) Meromorphic of the type $\frac{z^{2}}{w}=$ cte in neighborhoods of $M$ and $N$.

(ii) Holomorphic of the type $z^{6} \cdot w=$ cte in a neighborhood of $P_{1}(\alpha)$.

(iii) Holomorphic of the type $z^{3} \cdot w=c t e$ in a neighborhood of $P_{2}(\alpha)$.

In order to prove that the family has singularities of fixed analytic type, we will prove that:

(iv) If $\alpha \notin\left\{0,1, j, j^{2}, \infty\right\}$, then $\mathcal{F}_{\alpha}^{2}$ has a local meromorphic of the type $\frac{z^{3}}{w^{2}}=$ cte in neighborhoods of $J, K$ and $L$.

This follows from the fact that the characteristic numbers of $\mathcal{F}_{\alpha}^{2}$ at $J, K$ and $L$ are $2 / 3$ and $3 / 2$. In fact, the curve $Q$ has cuspidal singularities at these points, that is in a convenient local chart $(u, v)$ near one of them, $Q$ has an equation of the form $v^{2}-u^{3}=0$. As the reader can check, since $\mathcal{F}_{\alpha}^{2}$ is non-degenerate and $Q$ is invariant, this implies that the characteristic numbers are $2 / 3$ and $3 / 2$. Now, the Poincaré's linearization Theorem implies that $\mathcal{F}_{\alpha}^{2}$ is linearizable near $J, K$ and $L$, so that it has a local meromorphic first integral like in (iv). 
We have proved that the family $\left(\mathcal{F}_{\alpha}^{2}\right)_{\alpha \in \overline{\mathbb{C}} \backslash\left\{0,1, j, j^{2}, \infty\right\}}$ is a family of degree two with singularities of fixed analytic type. Since this family is obtained from $\left(\mathcal{F}_{\alpha}^{3}\right)_{\alpha \in \overline{\mathbb{C}}}$ by a Cremona transformation, it is not difficult to see that it satisfies also (b) and (c) of the main Theorem. We leave the details for the reader.

Remark 10. - We would like to observe that in a suitable affine coordinate system, the foliation $\mathcal{F}_{\alpha}^{2}$ is defined by the following differential equations:

$$
\mathcal{F}_{\alpha}^{2} \quad\left\{\begin{array} { l } 
{ \frac { d x } { d t } = 4 x - 9 x ^ { 2 } + y ^ { 2 } + \alpha ( 2 y - 4 x y ) , } \\
{ \frac { d y } { d t } = 6 y - 1 2 x y + 3 \alpha ( x ^ { 2 } - y ^ { 2 } ) , }
\end{array} \quad \text { or } \quad \mathcal { F } _ { \infty } ^ { 2 } \quad \left\{\begin{array}{l}
\frac{d x}{d t}=2 y-4 x y \\
\frac{d y}{d t}=3\left(x^{2}-y^{2}\right) .
\end{array}\right.\right.
$$

In this coordinate system, the line $R$ is the line at infinity and the quartic $Q$ is given by

$$
4 y^{2}(1-3 x)-4 x^{3}+\left(3 x^{2}+y^{2}\right)^{2}=0 .
$$

The finite singularities are $J=(1 / 2,-1 / 2), K=(0,0), L=(1 / 2,1 / 2)$ and $P_{1}(\alpha)=\left(\frac{4\left(\alpha^{2}+1\right)}{\left(\alpha^{2}+3\right)^{2}}\right.$, $\left.\frac{-8 \alpha}{\left(\alpha^{2}+3\right)^{2}}\right) \in Q$ and the singularities at the line $R$ are $M=[1: \sqrt{3} \mathrm{i}: 0], N=[1:-\sqrt{3} \mathrm{i}: 0]$ and $P_{2}(\alpha)=[1: \alpha: 0]$.

\section{Proof of the Corollary}

The families of degree greater than four are obtained by pulling back one of the families of $\S 2$. The idea is that, if $f: S \rightarrow T$ is a non-constant holomorphic map between two compact Riemann surfaces $S$ and $T$, where $T$ is an elliptic curve and $f$ has $k$ points of ramification, then

$$
\mathcal{X}(S) \leqslant-k
$$

where $\mathcal{X}(S)$ is the Euler characteristic of $S$. We observe that (22) follows from RiemannHurwitz formula. In fact, if the ramification points are $z_{1}, \ldots, z_{k}$ and the ramification number of $f$ at $z_{j}$ is $n_{j} \geqslant 2, j=1, \ldots, k$, then the Riemann-Hurwitz formula says that,

$$
\mathcal{X}(S)=d g(f) \cdot \mathcal{X}(T)-\sum_{j=1}^{k}\left(n_{j}-1\right)=-\sum_{j=1}^{k}\left(n_{j}-1\right) \leqslant-k,
$$

where $d g(f)$ is the topological degree of $f$.

\subsection{The families of degree $3 r-1, r \geqslant 2$}

Let us sketch how to obtain a family of degree $3 r-1, r \geqslant 2$, from the family of degree two. Consider homogeneous coordinates $(X, Y, Z)$ on $\mathbb{C}^{3}$ such that the invariant line $R$ for all $\mathcal{F}_{\alpha}^{2}$ is the line $(Z=0)$. Let $F: \mathbb{C P}(2) \rightarrow \mathbb{C P}(2)$ be a rational map which in this homogeneous coordinates in the target, is written as

$$
F(U, V, W)=\left(P(U, V, W), Q(U, V, W), W^{r}\right),
$$

where $P$ and $Q$ are homogeneous polynomials of degree $r$. We make the following generic assumptions on $P$ and $Q$ : 
(i) $\{(U, V) ; P(U, V, 0)=Q(U, V, 0)=0\}=\{(0,0)\}$. This condition implies that $F$ is well defined.

(ii) Let $\tilde{\Delta}=\frac{\partial P}{\partial U} \frac{\partial Q}{\partial V}-\frac{\partial P}{\partial V} \frac{\partial Q}{\partial U}$ and $\Delta=\Pi(\tilde{\Delta}=0)$, where $\Pi: \mathbb{C}^{3} \backslash\{0\} \rightarrow \mathbb{C P}(2)$ is the canonical projection. Then $\Delta$ is a smooth curve of degree $2 r-2$, transversal to the line $L_{\infty}=\Pi(W=0)$.

(iii) If $p_{o} \in \Delta \backslash L_{\infty}$, then $\operatorname{dim}_{\mathbb{C}}\left(\operatorname{ker}\left(d F\left(p_{o}\right)\right)=1\right.$.

(iv) The set $P=\left\{p_{o} \in \Delta \backslash L_{\infty} ; T_{p o} \Delta=\operatorname{ker}\left(d F\left(p_{o}\right)\right)\right\}$ is finite.

(v) If $p_{o} \in \Delta \backslash\left(P \cup L_{\infty}\right)$ and $\gamma:(\mathbb{C}, 0) \rightarrow\left(\mathbb{C P}(2), p_{o}\right)$ is a curve such that $0 \neq \gamma^{\prime}(0) \in \operatorname{ker}\left(d F\left(p_{o}\right)\right)$, then $(F \circ \gamma)^{\prime \prime}(0) \neq 0$.

In this case, there are holomorphic coordinate systems $\left(W_{1},(u, v)\right)$ and $\left(W_{2},(x, y)\right)$ around $p_{o}$ and $F\left(p_{o}\right)$, respectively, such that $u\left(p_{o}\right)=v\left(p_{o}\right)=0, x\left(F\left(p_{o}\right)\right)=y\left(F\left(p_{o}\right)\right)=0$ and $F(u, v)=\left(u, v^{2}\right)$. In these coordinates, we have $\Delta \cap W_{1}=(v=0)$ and $F(\Delta) \cap W_{2}=(y=0)$.

(vi) If $p_{o} \in P$ then there are holomorphic coordinate systems $\left(W_{1},(u, v)\right)$ and $\left(W_{2},(x, y)\right)$ around $p_{o}$ and $F\left(p_{o}\right)$, respectively, such that $u\left(p_{o}\right)=v\left(p_{o}\right)=0, x\left(F\left(p_{o}\right)\right)=y\left(F\left(p_{o}\right)\right)$ $=0$ and $F(u, v)=\left(u, \frac{1}{3} v^{3}-u v\right)$. In these coordinates, we have $\Delta \cap W_{1}=\left(u=v^{2}\right)$ and $F(\Delta) \cap W_{2}=\left(9 y^{2}=4 x^{3}\right)$.

(vii) Let $C=F(\Delta)$. Then $C$ has two types of singularities: the points in $F(P)$, which are singularities of cuspidal type; and singularities of nodal type. If $q_{o} \in C$ is a singularity of nodal type, then $F^{-1}\left(q_{o}\right) \cap \Delta$ contains two points, which are not on $P$.

Assumptions (ii)-(vii) are called Whitney's conditions. It is well-known that they are generic in the set of maps as in (23). We impose another condition in the points of $\Delta \cap L_{\infty}$. Observe that the critical points of the map $\left.F\right|_{L_{\infty}}: L_{\infty} \rightarrow L_{\infty}, F[u: v: 0]=[P(u, v, 0): Q(u, v, 0): 0]$, coincide with the critical points of the map $[P: Q]$.

(viii) The critical points of $[P: Q]: \overline{\mathbb{C}} \rightarrow \overline{\mathbb{C}}$ are of order two.

Let us denote by $C r$ the set of critical points of $\left.F\right|_{L_{\infty}}$. We observe that $C r=L_{\infty} \cap \Delta$. This follows from the fact that,

$$
\begin{aligned}
p_{o}=\left[1: v_{o}: 0\right] \in C r \Leftrightarrow 0 & =r\left(P \cdot \frac{\partial Q}{\partial v}-Q \cdot \frac{\partial P}{\partial v}\right)\left(p_{o}\right) \\
& =\frac{\partial P}{\partial u}\left(p_{o}\right) \frac{\partial Q}{\partial v}\left(p_{o}\right)-\frac{\partial P}{\partial v}\left(p_{o}\right) \frac{\partial Q}{\partial u}\left(p_{o}\right),
\end{aligned}
$$

where in the second equality, we have used Euler's identity. It is not difficult to see that, if $p_{o} \in C r$ then there are holomorphic coordinate systems $\left(W_{1},(v, w)\right)$ and $\left(W_{2},(x, y)\right)$ around $p_{o}$ and $F\left(p_{o}\right)$, respectively, such that, $v\left(p_{o}\right)=w\left(p_{o}\right)=0, x\left(F\left(p_{o}\right)\right)=y\left(F\left(p_{o}\right)\right)=0$ and $F(v, w)=\left(v^{2}+f(w), w^{r}\right)$, where $f$ is holomorphic.

Now, we take the quartic $Q$, which is invariant for all $\mathcal{F}_{\alpha}^{2}$, in such a way that:

(ix) The points $M$ and $N$ of tangency between $L_{\infty}=R$ and $Q$ are not in $C$ (see Fig. 7).

(x) $C$ cuts $Q$ transversely, so that the cuspidal points of $Q(J, K$ and $L)$ are not in $C \cup L_{\infty}$ and the singular points of $C$ are not in $Q \cup L_{\infty}$.

Let us consider the family of foliations $\left(\mathcal{G}_{\alpha}\right)_{\alpha \in \overline{\mathbb{C}}}$, where $\mathcal{G}_{\alpha}=F^{*}\left(\mathcal{F}_{\alpha}^{2}\right)$.

Lemma 2. - There exists a finite subset $F_{0} \subset \overline{\mathbb{C}}$, such that the family $\left(\mathcal{G}_{\alpha}\right)_{\alpha \in \overline{\mathbb{C}} \backslash F_{0}}$ is nondegenerate with singularities of fixed local analytic type. Moreover, the degree of any $\mathcal{G}_{\alpha}$ is $3 r-1$.

Proof. - Let $P_{1}(\alpha) \in Q$ and $P_{2}(\alpha) \in R$ be as in (ii) and (iii) of $\S 2.4$. Observe that the maps $\alpha \mapsto P_{1}(\alpha)$ and $\alpha \mapsto P_{2}(\alpha)$ are 1-1 parametrizations of $Q$ and $R$, respectively. We have seen that, if $\alpha \notin F_{1}:=\left\{0,1, j, j^{2}, \infty\right\}$, then all singularities of $\mathcal{F}_{\alpha}^{2}$ are non-degenerate. This means 
that $P_{1}\left(F_{1}\right)=\{J, K, L, M, N\}$ and $P_{2}\left(F_{1}\right) \supset\{M, N\}$. Define

$$
F_{2}=F_{1} \cup\left\{\alpha \in \overline{\mathbb{C}} ; P_{1}(\alpha) \in C \text { or } P_{2}(\alpha) \in C\right\} .
$$

It is not difficult to see that $F_{2}$ is finite. Now, observe that the topological degree of $F$ is $r^{2}$ and the set of critical values of $F$ is $C \cup R$. This implies that, if $p \in \mathbb{C P}(2) \backslash(C \cup R)$, then $F^{-1}(p)$ contains $r^{2}$ points. Moreover, for any $q \in F^{-1}(p), F$ is a biholomorphism in a neighborhood of $q$. It follows that, if $\alpha \notin F_{2}$, then $\mathcal{G}_{\alpha}$ has:

(a) $3 r^{2}$ non-degenerate singularities with a local meromorphic first integral of the type $\frac{z^{3}}{w^{2}}=$ cte, namely, the points in $F^{-1}(J) \cup F^{-1}(K) \cup F^{-1}(L)$.

(b) $r^{2}$ non-degenerate singularities with a local holomorphic first integral of the type $z^{6} . w=c t e$, namely, the points in $F^{-1}\left(P_{1}(\alpha)\right)$.

Now, the topological degree of $\left.F\right|_{L_{\infty}}: L_{\infty} \rightarrow R$ is $r$. This implies that if $p \in R \backslash C$, then $F^{-1}(p)$ contains $r$ points, all of them in $L_{\infty} \backslash \Delta$. It follows that if $\alpha \notin F_{2}$, then $F^{-1}(M) \cup F^{-1}(N)$ contains $2 r$ points, and $F^{-1}\left(P_{2}(\alpha)\right) r$ points. Let us study the singularities of $\mathcal{G}_{\alpha}$ in $F^{-1}\left\{M, N, P_{2}(\alpha)\right\}$.

Observe that, if $q \in L_{\infty} \backslash \Delta$, then there are holomorphic coordinate systems $\left(W_{1},(v, w)\right)$ and $\left(W_{2},(x, y)\right)$ around $q$ and $p=F(q)$, respectively, such that $v(q)=w(q)=0, x(p)=y(p)=0$, $W_{1} \cap L_{\infty}=(w=0), F(v, w)=\left(v, w^{r}\right)$ and $R \cap W_{2}=L_{\infty} \cap W_{2}=(y=0)$. Let us see how the foliation $\mathcal{G}_{\alpha}$ looks like in a neighborhood of a point $q \in L_{\infty} \backslash \Delta$. Since $R=(y=0)$ is invariant for $\mathcal{F}_{\alpha}^{2}$, this foliation can be defined in $W_{2}$ by a differential equation of the form

$$
\eta=y d x-(a+b x+c y+h . o . t) d y=0,
$$

where $a=0$ if, and only if $p$ is a singular point of $\mathcal{F}_{\alpha}^{2}$. If $\alpha \notin F_{2}$ and $p$ is a singular point of $\mathcal{F}_{\alpha}^{2}$, then $a=0$ and $b \neq 0$ (because $p$ is non-degenerate). It follows from (24) that $\mathcal{G}_{\alpha}$ is defined in $W_{1}$ by

$$
\begin{aligned}
& F^{*}(\eta)=w^{r} d v-(a+b v+\text { h.o.t. }) r w^{r-1} d w=0 \\
& \quad \Rightarrow w d v-r(a+b v+\text { h.o.t. }) d w=0 .
\end{aligned}
$$

This implies that, if $\alpha \notin F_{2}$ and $p$ is not a singularity of $\mathcal{F}_{\alpha}^{2}$, then $q$ is not a singularity of $\mathcal{G}_{\alpha}$. Moreover, if $p$ is a singularity of $\mathcal{F}_{\alpha}^{2}$, then $q$ is a non-degenerate singularity of $\mathcal{G}_{\alpha}$ and their characteristic numbers are $r b$ and $\frac{1}{r b}$. It follows that, if $\alpha \notin F_{2}$, then $\mathcal{G}_{\alpha}$ has:

(c) $2 r$ non-degenerate singularities on $L_{\infty}$ with a local meromorphic first integral of the type $\frac{w^{r}}{z^{2}}=$ cte. These singularities are the points of $F^{-1}(M) \cup F^{-1}(N)$.

(d) $r$ non-degenerate singularities on $L_{\infty}$ with a local holomorphic first integral of the type $w^{r} \cdot z^{3}=$ cte. These singularities are the points of $F^{-1}\left(P_{2}(\alpha)\right)$.

The proofs of (c) and (d) are based on Lemma 1 and are left for the reader. Let us consider now the points in $C r=\Delta \cap L_{\infty}$. If $\alpha \notin F_{2}$ and $q \in C r$, then $p=F(q)$ is not a singularity of $\mathcal{F}_{\alpha}^{2}$, so that (24) and the local form of $F$ in (viii) imply that $\mathcal{G}_{\alpha}$ can be defined in a neighborhood of $q$ by a differential equation of the type:

$$
\begin{aligned}
& w^{r} d\left(v^{2}+f(w)\right)-\left[a+b\left(v^{2}+f(w)\right)+c w^{r}+\cdots\right] r w^{r-1} d w=0 \\
& \quad \Rightarrow w d\left(v^{2}+f(w)\right)-r(a+\text { h.o.t. }) d w=0 .
\end{aligned}
$$

Therefore, $q$ is not a singularity of $\mathcal{G}_{\alpha}$.

It remains to study the singularities of $\mathcal{G}_{\alpha}$ on $\Delta_{1}:=\Delta \backslash L_{\infty}$. Let us define

$$
F_{3}=\left\{\alpha \in \overline{\mathbb{C}} ; \mathcal{G}_{\alpha} \text { has some degenerate singularity on } \Delta_{1}\right\}
$$


and $F_{0}=F_{2} \cup F_{3}$. In order to finish the proof of the lemma it is enough to prove that $F_{3}$ is finite.

Observe first that condition (x) implies that $C$ is not invariant for $\mathcal{F}_{\alpha}^{2}$. In fact, $Q$ is invariant for $\mathcal{F}_{\alpha}^{2}$ and $C \cap Q$ contains more than one point. If $C$ was an invariant for $\mathcal{F}_{\alpha}^{2}$ for some $\alpha$, then all points in $C \cap Q$ would be singular points of $\mathcal{F}_{\alpha}^{2}$. On the other hand, the unique singularity of $\mathcal{F}_{\alpha}^{2}$ in $Q \backslash\{J, K, L\} \cup L_{\infty}$ is $P_{1}(\alpha)$, which implies that one of the points of $C \cap Q$ is not a singularity of $\mathcal{F}_{\alpha}^{2}$.

Another fact that we will use is that $F\left(\Delta_{1}\right)=C_{1}:=C \backslash R$. Let us consider an affine coordinate system $(x, y) \in \mathbb{C}^{2}=\mathbb{C P}(2) \backslash R$, two polynomial vector fields $X$ and $Y$ such that $X_{\alpha}=X+\alpha Y$ defines $\mathcal{F}_{\alpha}^{2}$ in this system (see Remark 10) and a reduced polynomial $f$ such that $C_{1}=(f=0)$. Define $g_{\alpha}=X_{\alpha}(f)$ and $h_{\alpha}=X_{\alpha}\left(g_{\alpha}\right)$. The fact that $F_{3}$ is finite follows easily from the following assertions:

ASSERTION 1. - Let $\alpha \in \overline{\mathbb{C}}$ be such that $\mathcal{G}_{\alpha}$ has some degenerate singularity $q \in \Delta_{1}$. Then $p=F(q)$ satisfies $f(p)=g_{\alpha}(p)=h_{\alpha}(p)=0$.

Assertion 2. - The subset $\mathcal{A}$ of $\overline{\mathbb{C}} \times \mathbb{C}^{2}$ defined by

$$
\mathcal{A}=\left\{(\alpha, x, y) \in \overline{\mathbb{C}} \times \mathbb{C}^{2} ; f(x, y)=g_{\alpha}(x, y)=h_{\alpha}(x, y)=0\right\} \subset \overline{\mathbb{C}} \times C_{1}
$$

is algebraic of dimension zero.

Proof of Assertion 1. - We will prove that if $q \in \Delta_{1}$ is such that $F(q) \in C_{1}$ but $(\alpha, F(q)) \notin \mathcal{A}$ then, either $q$ is not a singularity of $\mathcal{G}_{\alpha}$, or $q$ is a non-degenerate singularity of $\mathcal{G}_{\alpha}$.

Let us observe first, that the conditions $f(p)=g_{\alpha}(p)=h_{\alpha}(p)=0$ depend only on the divisor $f$ and on the foliation $\mathcal{F}_{\alpha}^{2}$. In other words, if $U$ is an open set of $\mathbb{C}^{2}, Z_{\alpha}$ is a holomorphic vector field on $U$ which represents $\left.\mathcal{F}_{\alpha}^{2}\right|_{U}, f^{1} \in \mathcal{O}(U)$ is reduced and such that $C_{1} \cap U=\left(f^{1}=0\right)$ and $g_{\alpha}^{1}=Z_{\alpha}\left(f^{1}\right), h_{\alpha}^{1}=Z_{\alpha}\left(g_{\alpha}^{1}\right)$, then

$$
\left\{p \in U ; f^{1}(p)=g_{\alpha}^{1}(p)=h_{\alpha}^{1}(p)=0\right\}=\left\{p \in U ; f(p)=g_{\alpha}(p)=h_{\alpha}(p)=0\right\} .
$$

This follows from the fact that $Z_{\alpha}=u . X_{\alpha}$ and $f_{1}=v . f$ where $u, v \in \mathcal{O}^{*}(U)$, as the reader can check.

Let $q \in \Delta_{1}$ and $p=F(q) \in C_{1}$, so that $f(p)=0$. Suppose that $g_{\alpha}(p) \neq 0$. Since $g_{\alpha}=$ $X_{\alpha}(f)=d f\left(X_{\alpha}\right)$, it follows that $p$ is neither a singularity of $X_{\alpha}$ nor a singularity of $f$, so that $p \notin P$. Let us consider local coordinate systems $\left(W_{1},(u, v)\right)$ and $\left(W_{2},(z, w)\right)$ around $q$ and $p=F(q)$, respectively, such that $u(q)=v(q)=0, x(p)=y(p)=0$ and $F(u, v)=\left(u, v^{2}\right)$, like in (v). In these coordinates, we have $\Delta_{1} \cap W_{1}=(v=0)$ and $C_{1} \cap W_{2}=(w=0)$. The vector field $X$ can be written in the coordinate system $\left(W_{2},(z, w)\right)$ as $X_{\alpha}(z, w)=P(z, w) \frac{\partial}{\partial z}+$ $Q(z, w) \frac{\partial}{\partial w}$ and $\mathcal{F}_{\alpha}^{2}$ can be represented in $W_{2}$ by the 1 -form $\omega_{\alpha}=Q(z, w) d z-P(z, w) d w$. Since $C_{1} \cap W_{2}=(w=0)$ and $g_{\alpha}(p) \neq 0$, we must have $X_{\alpha}(w)(p)=Q(0,0) \neq 0$. Now, $\mathcal{G}_{\alpha}$ is represented in $W_{1}$ by

$$
\eta_{\alpha}:=F^{*}\left(\omega_{\alpha}\right)=Q\left(u, v^{2}\right) d u-P\left(u, v^{2}\right) 2 v d v
$$

so that $\eta_{\alpha}(0) \neq 0$ and $q$ is not a singular point of $\mathcal{G}_{\alpha}$.

Suppose now that $g_{\alpha}(p)=0$, but $h_{\alpha}(p) \neq 0$. Since $h_{\alpha}=X_{\alpha}\left(g_{\alpha}\right), p$ is not a singularity of $X_{\alpha}$, but it can be a singularity of $f$. We can have one of the following cases: (I) $p$ is not a singularity of $f$; (II) $p$ is a singularity of nodal type of $f$ and (III) $p$ is a cuspidal singularity of $f$. 
Case (I). In this case we can consider local coordinate systems $\left(W_{1},(u, v)\right)$ and $\left(W_{2},(z, w)\right)$ as before. Now, consider

$$
g_{\alpha}^{1}=X_{\alpha}(w)=Q(z, w)
$$

and

$$
h_{\alpha}^{1}=X_{\alpha}\left(g_{\alpha}^{1}\right)=P(z, w) Q_{z}(z, w)+Q(z, w) Q_{w}(z, w) .
$$

Since $g_{\alpha}(p)=0$ and $h_{\alpha}(p) \neq 0$ we must have $Q(0,0)=0$ and $P(0,0) Q_{z}(0,0) \neq 0$, so that $P(z, w)=a+$ h.o.t and $Q(z, w)=b z+c w+$ h.o.t., where $a, b \neq 0$. It follows from (25) that $\mathcal{G}_{\alpha}$ can be represented in a neighborhood of $q=(0,0)$ by a vector field of the form

$$
2 v \cdot P\left(u, v^{2}\right) \frac{\partial}{\partial u}+Q\left(u, v^{2}\right) \frac{\partial}{\partial v}=2 a v \frac{\partial}{\partial u}+b u \frac{\partial}{\partial v}+\text { h.o.t. }
$$

so that $q$ is a non-degenerate singularity of $\mathcal{G}_{\alpha}$.

Case (II). In this case, we have local charts $\left(W_{1},(u, v)\right)$ and $\left(W_{2},(z, w)\right)$ in neighborhoods of $q$ and $p$, as before. Moreover, we have that $f=w . f_{1}$, where $f_{1}(z, w)=a z+b w+$ h.o.t. where $a \neq 0$. On the other hand, a straightforward computation shows that $g_{\alpha}=Q \cdot f_{1}+w\left(P .\left(f_{1}\right)_{z}+\right.$ $\left.Q .\left(f_{1}\right)_{w}\right)$ and $h_{\alpha}(0)=Q(0,0)[2 a Q(0,0)+2 b P(0,0)] \neq 0$, so that $Q(0,0) \neq 0$. Therefore (25) implies that $q$ is not a singularity for $\mathcal{G}_{\alpha}$.

Case (III). In this case we take holomorphic coordinate systems $\left(W_{1},(u, v)\right)$ and $\left(W_{2},(z, w)\right)$ around $q$ and $p$, respectively, such that

$$
u(q)=v(q)=0, \quad z(p)=w(p)=0 \quad \text { and } \quad F(u, v)=\left(u, \frac{1}{3} v^{3}-u v\right) .
$$

In these coordinates, we have

$$
\Delta_{1} \cap W_{1}=\left(u=v^{2}\right) \quad \text { and } \quad C_{1} \cap W_{2}=\left(9 w^{2}-4 z^{3}=0\right) .
$$

If we set $f^{1}=9 w^{2}-4 z^{3}, g_{\alpha}^{1}=X_{\alpha}\left(f^{1}\right)$ and $h_{\alpha}^{1}=X_{\alpha}\left(g_{\alpha}^{1}\right)$, where $X_{\alpha}$ is as before, then

$$
h_{\alpha}^{1}(0,0)=18(Q(0,0))^{2} \Longrightarrow Q(0,0) \neq 0,
$$

as the reader can check. Therefore, if $\omega_{\alpha}=Q(z, w) d z-P(z, w) d w$, then

$$
\eta_{\alpha}=F^{*}\left(\omega_{\alpha}\right)=[Q \circ F+v P \circ F] d u+P \circ F \cdot\left[u-3 v^{2}\right] d v \Longrightarrow \eta_{\alpha}(q)=Q(0,0) d u \neq 0 .
$$

Therefore $q$ is not a singularity of $\mathcal{G}_{\alpha}$.

Proof of Assertion 2. - Since $f, g_{\alpha}$ and $h_{\alpha}$ are polynomials in the three variables $(\alpha, x, y)$, it is clear that $\mathcal{A}$ is an algebraic subset of $\overline{\mathbb{C}} \times \mathbb{C}^{2}$. In order to prove that it has dimension zero, it is sufficient to show that their points are isolated. Fix a point $\left(\alpha_{0}, p_{0}\right)=\left(\alpha_{0}, x_{0}, y_{0}\right) \in \mathcal{A}$. Suppose first that $p_{0}$ is not a singularity of $C_{1}$. Observe that there exists $\alpha_{1} \neq \alpha_{o}$ such that $\mathcal{F}_{\alpha_{1}}^{2}$ is transversal to $C_{1}$ at $p_{0}$. Let $X$ and $Y$ be two polynomial vector fields on $\mathbb{C}^{2}$ which represent $\mathcal{F}_{\alpha_{0}}^{2}$ and $\mathcal{F}_{\alpha_{1}}^{2}$, respectively, and such that $\mathcal{F}_{\alpha}^{2}$ is represented by $X_{\alpha}=\left(\alpha-\alpha_{1}\right) X+\left(\alpha-\alpha_{0}\right) Y$. Since $p_{0}$ is a smooth point of $C_{1}$ and $Y$ is transversal to $C_{1}$ at $p_{0}$, there exists a local coordinate system $(W,(u, v))$ around $p_{0}$ such that $u\left(p_{0}\right)=v\left(p_{0}\right)=0, W \cap C_{1}=\left(f^{1}=v=0\right)$ and $Y(u, v)=\frac{\partial}{\partial v}$. In this coordinate system $X$ can be written as

$$
X(u, v)=A(u, v) \frac{\partial}{\partial u}+(k(u)+v \ell(u, v)) \frac{\partial}{\partial v} .
$$


Since $C_{1}$ is not invariant for $\mathcal{F}_{\alpha_{0}}$, we must have $k(u) \not \equiv 0$. Moreover, $A(u, 0) \not \equiv 0$, because $Y$ is transversal to $X$ in almost all points of $C_{1} \cap W=(v=0)$. Let $g_{\alpha}^{1}=X_{\alpha}\left(f^{1}\right)=X_{\alpha}(v)$ and $h_{\alpha}^{1}=X_{\alpha}\left(g_{\alpha}^{1}\right)$, so that $\mathcal{A} \cap(\overline{\mathbb{C}} \times W)=\left(v=g_{\alpha}^{1}=h_{\alpha}^{1}=0\right)$. Now, a straighforward computation shows that

and

$$
g_{\alpha}^{1}(u, v)=\alpha-\alpha_{0}+\left(\alpha-\alpha_{1}\right)(k(u)+v \ell(u, v))
$$

$$
h_{\alpha}^{1}(u, 0)=\left(\alpha-\alpha_{1}\right)\left[\left(\alpha-\alpha_{1}\right)\left(A(u, 0) k^{\prime}(u)+k(u) \ell(u, 0)\right)+\left(\alpha-\alpha_{0}\right) \ell(u, 0)\right] .
$$

Therefore, the set $\mathcal{A}$ can be defined in $\overline{\mathbb{C}} \times W$ by $g_{\alpha}^{1}(u, 0)=h_{\alpha}^{1}(u, 0)=0 \Longrightarrow$

$$
\alpha-\alpha_{0}+\left(\alpha-\alpha_{1}\right) k(u)=\left(\alpha-\alpha_{1}\right)^{2} \cdot A(u, 0) \cdot k^{\prime}(u)=0,
$$

as the reader can check. Since $\left(\alpha_{0}-\alpha_{1}\right) k(0)=g_{\alpha_{0}}^{1}(0,0)=0$, the function $k$ is non-constant, so that $k^{\prime}(u) \not \equiv 0$. This fact together with (26) imply that $\left(\alpha_{0}, p_{0}\right)$ is an isolated point of $\mathcal{A}$.

The above argument implies that, if $S$ is the set of singularities of $C_{1}$, then all irreducible components of $\mathcal{A}$ in $\overline{\mathbb{C}} \times\left(C_{1} \backslash S\right)$ have dimension zero. Since $S$ is finite, if $\mathcal{A}$ contains some irreducible component, say $B$, of dimension greater than one, then $B=\overline{\mathbb{C}} \times\{p\}$, where $p \in S$. However, as the reader can verify in the proofs of cases (II) and (III) in Assertion 1, we have the following:

(II.1) If $p$ is a nodal point of $C_{1}$ then $\mathcal{A} \cap(\overline{\mathbb{C}} \times\{p\})$ contains exactly two points: the points $(\alpha, p)$ such that $X_{\alpha}(p)$ is tangent to one of the branchs of $C_{1}$ at $p$.

(III.1) If $p$ is a cuspidal point of $C_{1}$ then $\mathcal{A} \cap(\overline{\mathbb{C}} \times\{p\})$ contains exactly one point: the point $(\alpha, p)$ such that $X_{\alpha}(p)$ is in the direction of the tangent cone of $C_{1}$ at $p$.

We leave the details of the proof of these facts for the reader. This finishes the proof of Assertion 2.

Now, let $\alpha \notin F_{0}$. It follows from the above arguments that all singularities of $\mathcal{G}_{a}$ are nondegenerate as in (a), (b), (c), (d) or

(e) Singularities of $\mathcal{G}_{a}$ on $\Delta_{1}$. The foliation $\mathcal{G}_{\alpha}$ has a local first integral of the type $z . w=c t e$ in a neighborhood of each singularity of this type.

In fact, if $q \in \Delta_{1}$ is a singularity of $\mathcal{G}_{\alpha}$, as we have seen in the proof of case (I) in Assertion 1, the linear of a vector field which represents $\mathcal{G}_{\alpha}$ in a neighborhood of $q$, can be written in a suitable coordinate system as $2 a v \frac{\partial}{\partial u}+b u \frac{\partial}{\partial v}$, where $a, b \neq 0$. This implies that the characteristic numbers of $\mathcal{G}_{\alpha}$ at $q$ are both -1 . On the other hand, since $p=F(q)$ is not a singularity for $\mathcal{F}_{\alpha}^{2}$, this foliation has a local regular holomorphic first integral, say $h$, in a neighborhood of $p$. It follows that $h \circ F$ is a local holomorphic first integral for $\mathcal{G}_{\alpha}$. Since the characteristic numbers of $\mathcal{G}_{\alpha}$ at $q$ are both -1 , this first integral is of Morse type, so that it can be written in a suitable coordinate system as $z \cdot w=$ cte.

It remains to prove that $\mathcal{G}_{\alpha}$ has degree $3 r-1$. We will use Baum-Bott formula in the proof (cf. [4] and [3]). The Baum-Bott index of a foliation $\mathcal{F}$ in a non-degenerate singularity $p$ is defined as follows. Let $Z$ be a holomorphic vector field which represents $\mathcal{F}$ in a neighborhood of $p$ and $A=D Z(p)$. The number $\frac{\operatorname{tr}(A)^{2}}{\operatorname{det}(A)}$ does not depend on the vector field $Z$, representing $\mathcal{F}$ near $p$. Define the Baum-Bott index of $\mathcal{F}$ at $p$ by

$$
B B(\mathcal{F}, p)=\frac{\operatorname{tr}(A)^{2}}{\operatorname{det}(A)} .
$$

In particular, if the characteristic numbers of $\mathcal{F}$ at $p$ are $a$ and $a^{-1}$, then $B B(\mathcal{F}, p)=a+a^{-1}+2$. We would like to observe that the Baum-Bott index can be defined for any isolated singularity 
of $\mathcal{F}$ (cf [4]), but we will use it only in the non-degenerate case. The Baum-Bott formula in $\mathbb{C P}(2)$ can be stated as follows (cf. [17]): let $\mathcal{F}$ be a foliation of degree $d$ in $\mathbb{C P}(2)$. Then

$$
\sum_{p \in \operatorname{sing}(\mathcal{F})} B B(\mathcal{F}, p)=(d+2)^{2}
$$

In our case, if $\alpha \notin F_{0}$, then the singularities of $\mathcal{G}_{\alpha}$ are the following:

Case (a). $3 r^{2}$ singularities of index $\frac{(2+3)^{2}}{2 \times 3}=\frac{25}{6}$.

Case (b). $r^{2}$ singularities of index $\frac{(1-6)^{2}}{-6 \times 1}=-\frac{25}{6}$.

Case (c). $2 r$ singularities of index $\frac{(2+r)^{2}}{2 \times r}=\frac{(r+2)^{2}}{2 r}$.

Case (d). $r$ singularities of index $\frac{(3-r)^{2}}{-r \times 3}=-\frac{(r-3)^{2}}{3 r}$.

Case (e). The singularities like in (e) have Baum-Bott index zero.

This implies that:

$$
\begin{aligned}
\sum_{p \in \operatorname{sing}\left(\mathcal{G}_{\alpha}\right)} B B(\mathcal{F}, p) & =\frac{25}{6} \times 3 r^{2}-\frac{25}{6} \times r^{2}+\frac{(r+2)^{2}}{2 r} \times 2 r-\frac{(r-3)^{2}}{3 r} \times r \\
& =(3 r+1)^{2}=(3 r-1+2)^{2} .
\end{aligned}
$$

Therefore $d g\left(\mathcal{G}_{\alpha}\right)=3 r-1$, which proves the lemma.

We now finish the proof of the Corollary for this type of family.

LEMMA 3. - Let $E$ be as in the main Theorem. If $\alpha \in E$, then the foliation $\mathcal{G}_{a}$ has a rational first integral, say of degree $d_{\alpha}$. Moreover, for any $k \in \mathbb{N}$, the sets $\left\{\alpha \in E ; d_{\alpha} \leqslant k\right\}$ and

$$
\left\{\alpha \in E ; \text { the genus of the general integral curve of } \mathcal{F}_{\alpha} \text { is } \leqslant k\right\}
$$

are finite.

Proof. - If $\alpha \in E$ then $\mathcal{F}_{\alpha}^{2}$ has a rational first integral, say $\widehat{R}_{\alpha}$, where the general level surface $\widehat{R}_{\alpha}=$ cte is irreducible of degree $\hat{d}_{\alpha}$. Observe that the rational map $R_{\alpha}=\widehat{R}_{\alpha} \circ F$ is a first integral of $\mathcal{G}_{\alpha}$, whose general level $R_{\alpha}=$ cte is irreducible. This implies that the degree of the general level surface $R_{\alpha}=$ cte is $d_{\alpha}=r^{2} . \hat{d}_{\alpha}$. Therefore the set $\left\{\alpha \in E ; d_{\alpha}<k\right\}$ is finite for all $k>0$.

Now consider an irreducible level surface $L_{c}=\left(R_{\alpha}=c\right)$ such that $L_{c}$ does not contain any non-dicritical singularity of $\mathcal{G}_{\alpha}$ and $F\left(L_{c}\right)$ does not contain any singular point of $C$. Let $\hat{L}_{c}=F\left(L_{c}\right)$. Since $L_{c}$ is irreducible, we must have $L_{c}=F^{-1}\left(\hat{L}_{c}\right)$, so that the topological degree of $\left.F\right|_{L_{c}}: L_{c} \rightarrow \hat{L}_{c}$ is $r^{2} \geqslant 4$. Observe that $\hat{L}_{c}$ is transversal to $C$. In fact, if it was not transversal to $C$ it would be tangent to $C$ in a point $p \in \hat{L}_{c} \cap C$. This point is not a singular point of $C$, by assumption. Therefore $p$ must be a tangency in a regular point of $C$, so that $F^{-1}(p)$ contains some non-dicritical singularity of $\mathcal{G}_{\alpha}$ (see case (I) in the proof of Assertion 2 in Lemma 2), which contradicts the choice of $L_{c}$. This implies that $\hat{L}_{c} \cap C$ contains $\delta . \hat{d}_{\alpha}$ points, $\delta=d g(C)$. Now, for each point $p \in \hat{L}_{c} \cap C$, let $q(p) \in \Delta$ be such that $p=F(q(p))$. It is clear that the point $q(p) \in L_{c}$ is a ramification point of $\left.F\right|_{L_{c}}$, so that $\left.F\right|_{L_{c}}$ has at least $\delta . \hat{d}_{\alpha}$ ramification points. This implies that, if $\tilde{L}_{c}$ is the normalization of $L_{c}$, then $\mathcal{X}\left(\tilde{L}_{c}\right) \leqslant-\delta . \hat{d}_{\alpha}$, so that $g\left(L_{c}\right) \geqslant 1+\frac{\delta . \hat{d}_{\alpha}}{2}$. It follows that $\left\{\alpha \in E\right.$; the genus of the general integral curve of $\mathcal{F}_{\alpha}$ is $\left.\leqslant k\right\}$ is finite for any $k>0$.

This finishes the proof of the Corollary for families of degrees of the form $3 r-1, r \geqslant 2$. In order to obtain families of degrees of the form $3 r$ and $3 r+1$ we can use the same type of 
construction, but now using the families of degrees 3 and 4 , respectively. In what follows, we will sketch how these families can be constructed. Since the proof of the Corollary for them is analogous to the previous case, we will only say how is the map $F: \mathbb{C P}(2) \rightarrow \mathbb{C P}(2)$ and how are the singularities of the families.

\subsection{The families of degree $3 r, r \geqslant 2$}

Consider the family $\left(\mathcal{F}_{\alpha}^{3}\right)_{\alpha \in \overline{\mathbb{C}}}$. Let $\ell_{1}$ and $\ell_{2}$ be two of the straight lines invariant for all foliations of the family. Consider homogeneous coordinates $(X, Y, Z) \in \mathbb{C}^{3}$ such that $\ell_{1}=\Pi(Y=0)$ and $\ell_{2}=\Pi(Z=0)$. Take $F: \mathbb{C P}(2) \rightarrow \mathbb{C P}(2)$, which in homogeneous coordinates is of the form $(X, Y, Z)=F(U, V, W)=\left(P(U, V, W), V^{r}, W^{r}\right)$, where $P$ is a homogeneous polynomial of degree $r$. Let $\Delta=\Pi\left(\left\{(U, V, W) ; \frac{\partial P}{\partial U}(U, V, W)=0\right\}\right), L_{1}=(V=0), L_{2}=(W=0)$, and $C=F(\Delta)$, so that the set of critical points of $F$ is $\Delta \cup L_{1} \cup L_{2}, F\left(L_{1}\right)=\ell_{1}$ and $F\left(L_{2}\right)=\ell_{2}$. We suppose that $P$ satisfies the following generic properties:

(i.1) $\{U ; P(U, 0,0)=0\}=\{0\}$. This implies that the map $F$ is well defined and that $[1: 0: 0]=L_{1} \cap L_{2} \notin \Delta$.

(ii.1) The curve $\Delta$ is smooth of degree $r-1$ and transversal to $L_{1} \cup L_{2}$.

(iii.1) $F$ satisfies Whitney's conditions in $\mathbb{C P}(2) \backslash\left(L_{1} \cup L_{2}\right)$ (see conditions (iii)-(vii) in $\$ 3.1$ ).

(iv.1) The critical points of the polynomials $U \mapsto P(U, 1,0)$ and $V \mapsto P(U, 0,1)$ are of order two (see condition (viii) in $\$ 3.1$ ).

(v.1) The curve $C$ is transversal to the curve $C_{1} \cup C_{2} \cup \ell_{0} \cup \ell_{1} \cup \ell_{2}$. In particular the dicritical singularities of the generic foliation $\mathcal{F}_{\alpha}^{3}, p_{01}, p_{02}, p_{12}, p_{0}, p_{1}, p_{2}, q_{1}$ and $q_{2}$ are not in the curve $C$.

We define $\mathcal{H}_{\alpha}=F^{*}\left(\mathcal{F}_{\alpha}^{3}\right)$. It can be proved that there exists a finite set $H_{0}$ such that, if $\alpha \notin H_{0}$ then all singularities of $\mathcal{H}_{\alpha}$ are non-degenerate of the following types:

(a.1) One radial singularity at the point $p=[1: 0: 0]=F^{-1}\left(p_{12}\right)$. In this case $\mathcal{H}_{\alpha}$ has a local meromorphic first integral of the type $\frac{w}{z}=$ cte in a neighborhood of $p$ and Baum-Bott index 4.

(b.1) $3 r^{2}$ singularities at $F^{-1}\left\{q_{1}, q_{2}, p_{0}\right\}$ with Baum-Bott indexes $\frac{9}{2}$. In this case $\mathcal{H}_{\alpha}$ has a local meromorphic first integral of the type $\frac{w^{2}}{z}=c t e$ in a neighborhood of each of these points.

(c.1) $2 r$ singularities at $F^{-1}\left(\left\{p_{01}, p_{02}\right\}\right)$ with Baum-Bott indexes $\frac{(r+1)^{2}}{r}$. In this case $\mathcal{H}_{\alpha}$ has a local meromorphic first integral of the type $\frac{w^{r}}{z}=$ cte in a neighborhood of each of these points.

(d.1) $2 r$ singularities at $F^{-1}\left(\left\{p_{1}, p_{2}\right\}\right)$ with Baum-Bott indexes $\frac{(r+2)^{2}}{2 r}$. In this case $\mathcal{H}_{\alpha}$ has a local meromorphic first integral of the type $\frac{w^{r}}{z^{2}}=c t e$ in a neighborhood of each of these points.

(e.1) $2 r$ singularities at $F^{-1}\left(\left\{Q_{1}(\alpha), Q_{2}(\alpha)\right\}\right)$ with Baum-Bott indexes $-\frac{(r-3)^{2}}{3 r}$. In this case $\mathcal{H}_{\alpha}$ has a local holomorphic first integral of the type $w^{r} . z^{3}=c t e$ in a neighborhood of each of these points.

(f.1) $2 r^{2}$ singularities at $F^{-1}\left(\left\{P_{2}(\alpha), Q_{0}(\alpha)\right\}\right)$ with Baum-Bott indexes $-\frac{4}{3}$. In this case $\mathcal{H}_{\alpha}$ has a local holomorphic first integral of the type $w^{3} . z=c t e$ in a neighborhood of each of these points.

(g.1) $r^{2}$ singularities at $F^{-1}\left(P_{1}(\alpha)\right)$ with Baum-Bott indexes $-\frac{25}{6}$. In this case $\mathcal{H}_{\alpha}$ has a local holomorphic first integral of the type $w^{6} . z=c t e$ in a neighborhood of each of these points. 
(h.1) The remaining singularities correspond to the points of tangency between $\mathcal{F}_{\alpha}^{3}$ and $C$. They have Baum-Bott index 0 and $\mathcal{H}_{\alpha}$ has a local holomorphic first integral of the type $w . z=$ cte in a neighborhood of each of these points.

The foliation $\mathcal{H}_{\alpha}$ has degree $3 r$ because:

$$
\begin{aligned}
4 & +3 r^{2} \times \frac{9}{2}+2 r \times \frac{(r+1)^{2}}{r}+2 r \times \frac{(r+2)^{2}}{2 r}-2 r \times \frac{(r-3)^{2}}{3 r} \\
& -2 r^{2} \times \frac{4}{3}-r^{2} \times \frac{25}{6}=(3 r+2)^{2} .
\end{aligned}
$$

\subsection{The families of degree $3 r+1, r \geqslant 2$}

Consider the family $\left(\mathcal{F}_{\alpha}^{4}\right)_{\alpha \in \overline{\mathbb{C}}}$. Let $\ell_{1}, \ell_{2}$ and $\ell_{3}$ be three of the straight lines invariant for all foliations of the family, such that $\ell_{1} \cap \ell_{2} \neq \ell_{1} \cap \ell_{3}$. Consider homogeneous coordinates $(X, Y, Z) \in \mathbb{C}^{3}$ such that $\ell_{1}=\Pi(X=0), \ell_{2}=\Pi(Y=0)$ and $\ell_{3}=\Pi(Z=0)$. Take $F: \mathbb{C P}(2) \rightarrow \mathbb{C P}(2)$, which in homogeneous coordinates is of the form

$$
(X, Y, Z)=F(U, V, W)=\left(U^{r}, V^{r}, W^{r}\right) .
$$

Let $\mathcal{K}_{\alpha}=F^{*}\left(\mathcal{F}_{\alpha}^{4}\right)$. It can be proved that if $\alpha \notin\left\{1, j, j^{2}, \infty\right\}$ then all singularities of $\mathcal{K}_{\alpha}$ are non-degenerate of the following types:

(a.2) $3 r^{2}+3$ radial singularities. The foliation $\mathcal{K}_{\alpha}$ has Baum-Bott index 4 and a local meromorphic first integral of the type $\frac{w}{z}=$ cte in a neighborhood of each of these points.

(b.2) $6 r$ singularities with Baum-Bott indexes $\frac{(r+1)^{2}}{r}$ and a local meromorphic first integral of the type $\frac{w^{r}}{z}=$ cte in a neighborhood of each of these points. This set of points is $F^{-1}\left(\left\{p_{1}, \ldots, p_{6}\right\}\right)$, where $p_{1}, \ldots, p_{6}$ are the six radial singularities contained in $\bigcup_{i=1}^{3} \ell_{i}$ which are not in the intersection of any two of these lines.

(c.2) $3 r$ singularities with Baum-Bott indexes $-\frac{(r-3)^{2}}{3 r}$ and a local holomorphic first integral of the type $w^{r} . z^{3}=$ cte in a neighborhood of each of these points.

(d.2) $6 r^{2}$ singularities with Baum-Bott indexes $-4 / 3$ and a local holomorphic first integral of the type $w^{3} . z=c t e$ in a neighborhood of each of these points.

Therefore,

$$
\begin{aligned}
& \sum_{p \in \operatorname{sing}\left(\mathcal{K}_{\alpha}\right)} B B\left(\mathcal{K}_{\alpha}, p\right) \\
& =\left(3 r^{2}+3\right) \times 4+6 r \times \frac{(r+1)^{2}}{r}-3 r \times \frac{(r-3)^{2}}{3 r}-6 r^{2} \times \frac{4}{3}=(3 r+3)^{2},
\end{aligned}
$$

which implies that $d g\left(\mathcal{K}_{\alpha}\right)=3 r+1$.

We observe that the set of singular points of $F$ is $(U=0) \cup(V=0) \cup(W=0)$ and its image by $F$ is $\ell_{1} \cup \ell_{2} \cup \ell_{3}$, which is invariant for all foliations $\mathcal{F}_{\alpha}^{4}$. In particular, there are no singularities which come from tangencies, like in (e) or (h.1).

We remark also that, if $L$ is a leaf of $\mathcal{K}_{\alpha}$ such that the leaf of $\mathcal{F}_{\alpha}^{4}, F(L):=\hat{L}$, is not contained in the set $\mathcal{L}$ of straight lines invariant for $\mathcal{F}_{\alpha}^{4}$, then the "ramification points" of $\left.F\right|_{L}: L \rightarrow \hat{L}$ are "contained" in the dicritical points of type (b.2). In order to precise this sentence, we must solve by blowing-up these dicritical points. Let us give an idea. Fix a point $q \in F^{-1}\left(p_{1}\right)$, for instance, where $p_{1} \in \ell_{1}$ and $q \in L_{1}$. Then there are holomorphic coordinate systems $\left(W_{1},(u, v)\right)$ and $\left(W_{2},(x, y)\right)$ around $q$ and $p_{1}$ respectively, such that $u(q)=v(q)=0, x\left(p_{1}\right)=y\left(p_{1}\right)=0$, $W_{1} \cap L_{1}=(v=0), W_{2} \cap \ell_{1}=(y=0)$ and $(x, y)=F(u, v)=\left(u, v^{r}\right)$. Since $p_{1}$ is radial and 
$(y=0)$ is invariant for $\mathcal{F}_{\alpha}^{4}$, this foliation can be defined in a neighborhood of $p_{1}$ by a differential equation of the form

$$
\eta=y d x-(x+A(x, y)) d y=0,
$$

where the order of $A$ at $(0,0)$ is at least two. This implies that $\mathcal{K}_{\alpha}$ is defined by $\omega=\frac{1}{v^{r-1}} F^{*}(\eta)$, which can be written as

$$
\omega=v d u-\left(r u+u^{2} A_{1}\left(u, v^{r}\right)+u v^{r} A_{2}\left(u, v^{r}\right)+v^{2 r} A_{3}\left(u, v^{r}\right)\right) d v=0,
$$

as the reader can check. Therefore, the resolution of $\mathcal{K}_{\alpha}$ at $q$ involves $r$ blowing-ups in which a dicritical divisor appears at the last blowing-up. Let us denote by $\pi$ the composition of these blowing-ups and by $D$ the last divisor. One of the charts of $D$ can be written as $(t, v)$, where $\pi(t, v)=\left(t v^{r}, v\right)$. Observe that $\pi^{*}\left(\mathcal{K}_{\alpha}\right)$ is defined in this chart by $\frac{1}{v^{r+1}} \pi^{*}(\omega)$, which can be written as

$$
d t-v^{r-1}\left(t^{2} A_{1}\left(t v^{r}, v\right)+t A_{2}\left(t v^{r}, v^{r}\right)+A_{3}\left(t v^{r}, v^{r}\right)\right) d v=0,
$$

so that the foliation is transversal to the divisor $D=(v=0)$. Let us consider now the chart $(s, y)$ of the blowing-up $\pi_{1}$ at $p_{1}$ such that $\pi_{1}(s, y)=(s y, y)$. If $F_{1}$ is the rational map such that $\pi_{1} \circ F_{1}=F \circ \pi$, then it is not difficult to see that $F_{1}(t, v)=\left(t, v^{r}\right)$, so that $F_{1}$ ramifies in order $r$ along $D$. This procedure provides us the "points of ramification" of $\left.F\right|_{L}$ needed to use (22) and to prove the analogous of Lemma 3 for the family $\mathcal{K}_{\alpha}$.

\section{REFERENCES}

[1] Ahlfors L.V., S Ario L., Riemann Surfaces, Princeton, 1960.

[2] ARnold V., Chapitres supplémentaires de la théorie des équations différentielles ordinaires, Éditions MIR, 1980.

[3] BAUM P., BотT R., On the zeroes of meromorphic vector fields, in: Essais en l'honneur de De Rham, 1970, pp. 29-47.

[4] Brunella M., Feuilletages holomorphes sur les surfaces complexes compactes, Ann. Scient. Éc. Norm. Sup., 4e série 30 (1997) 569-594.

[5] Brunella M., Courbes entières et feuilletages holomorphes, Enseig. Math. 45 (1-2) (1999) 196216.

[6] Camacho C., Lins Neto A., Geometric Theory of Foliations, Birkhäuser, 1985.

[7] CAmpillo A., CARnicer M., Proximity inequalities and bounds for the degree of invariant curves by foliations in $\mathcal{P}_{\mathbb{C}}^{2}$, Trans. Amer. Math. Soc. 349 (9) (1997) 2211-2228.

[8] Carnicer M., The Poincaré problem in the non-dicritical case, Ann. de Math. 140 (1994) 289-294.

[9] Cerveau D., Lins Neto A., Holomorphic foliations in $\mathbb{C} P(2)$ having an invariant algebraic curve, Ann. Inst. Fourier 41 (4) (1991) 883-903.

[10] Cerveau D., Mattei J.F., Formes intégrables holomorphes singulières, Astérisque 97 (1982).

[11] Darboux G., Mémoire sur les équations différentielles algébriques du premier ordre et du premier degré (Mélanges), Bull. Sci. Math. (2) 2 (1878) 60-96, 123-144, 151-200.

[12] Ehresmann C., Reeb G., Les connexions infinitésimales dans un espace fibré différentiable, in: Colloque de topologie, Bruxelles, 1950, pp. 29-55.

[13] Godbillon C., Études géométriques I, Université Louis Pasteur, 1985.

[14] InsE E.L., Ordinary Differential Equations, Dover Books on Advanced Mathematics.

[15] Jounnolou J.-P., Équations de Pfaff algébriques, Lecture Notes in Math., Vol. 708, Springer-Verlag, Berlin, 1979.

[16] LimA E.L., Grupo Fundamental e espaços de recobrimento, Projeto Euclides, IMPA, 1993.

[17] Lins Neto A., Algebraic solutions of polynomial differential equations and foliations in dimension two, in: Lect. Notes Math., Vol. 1345, pp. 192-231. 
[18] Mattei J.F., Moussu R., Holonomie et intégrales premières, Ann. Sc. Ec. Norm. Sup. 13 (1980) 469-523.

[19] Moulin Ollagnier J., About a conjecture on quadratic vector fields, Preprint École Polytechnique, Paris, 2000.

[20] Painlevé P., "Sur les intégrales algébriques des équations différentielles du premier ordre” and "Mémoire sur les équations différentielles du premier ordre", Oeuvres de Paul Painlevé; Tome II, Éditions du Centre National de la Recherche Scientifique, 15, quai Anatole-France, 75700, Paris, 1974.

[21] Poincaré H., Sur l'intégration algébrique des équations différentielles, C. R. Acad. Sci. 112 (1891) 761-764.

[22] PoINCARÉ H., Sur l'intégration algébrique des équations différentielles du premier ordre et du premier degré, Rendiconti del Circolo Matemático di Palermo 5 (1891) 161-191.

[23] PoInCARÉ H., Sur l'intégration algébrique des équations différentielles du premier ordre et du premier degré, Rendiconti del Circolo Matemático di Palermo 11 (1897) 193-239.

(Manuscript received September 6, 2000; accepted, after revision, June 13, 2001.)

Alcides Lins Neto

Instituto de Matemática Pura e Aplicada,

Estrada Dona Castorina, 110, Horto,

Rio de Janeiro, Brazil

E-mail: alcides@impa.br 